\title{
HIGH RESOLUTION IMAGING OF VERY LOW MASS SPECTRAL BINARIES: THREE RESOLVED SYSTEMS AND DETECTION OF ORBITAL MOTION IN AN L/T TRANSITION BINARY*
}

\author{
Daniella C. Bardalez Gagliuffi ${ }^{1,2,4}$, Christopher R. Gelino ${ }^{2,3}$, and Adam J. Burgasser ${ }^{1}$ \\ ${ }^{1}$ Center for Astrophysics and Space Sciences, University of California, San Diego, 9500 Gilman Dr., Mail Code 0424, La Jolla, CA 92093, USA \\ ${ }^{2}$ Infrared Processing and Analysis Center, California Institute of Technology, Pasadena, CA 91125, USA \\ ${ }^{3}$ NASA Exoplanet Science Institute, California Institute of Technology, Pasadena, CA 91125, USA \\ Received 2015 August 31; accepted 2015 September 29; published 2015 November 2
}

\begin{abstract}
We present high resolution Laser Guide Star Adaptive Optics imaging of 43 late-M, L and T dwarf systems with Keck/NIRC2. These include 17 spectral binary candidates, systems whose spectra suggest the presence of a T dwarf secondary. We resolve three systems: 2MASS J1341-3052, SDSS J1511+0607 and SDSS J2052-1609; the first two are resolved for the first time. All three have projected separations $<8$ AU and estimated periods of 14-80 years. We also report a preliminary orbit determination for SDSS J2052-1609 based on six epochs of resolved astrometry between 2005 and 2010. Among the 14 unresolved spectral binaries, 5 systems were confirmed binaries but remained unresolved, implying a minimum binary fraction of $47_{-11}^{+12} \%$ for this sample. Our inability to resolve most of the spectral binaries, including the confirmed binaries, supports the hypothesis that a large fraction of very low mass systems have relatively small separations and are missed with direct imaging.
\end{abstract}

Key words: binaries: general - brown dwarfs - stars: fundamental parameters - stars: individual (2MASS

J13411160-30525049, SDSS J151114.66+060742.9, SDSS J205235.31-160929.8) - stars: low-mass

\section{INTRODUCTION}

Observational studies of field brown dwarfs indicate that only $\sim 10 \%-20 \%$ are found in very low mass (VLM) binary systems (Bouy et al. 2003; Close et al. 2003; Basri \& Reiners 2006; Allen 2007; Burgasser 2007a; Kraus \& Hillenbrand 2012). In contrast, the binary fraction for $\mathrm{G}$ stars is $\sim 40 \%$ (Duquennoy \& Mayor 1991) and 30\% for M dwarfs (Fischer \& Marcy 1992). These statistics suggest a steady decline of binary fraction with mass. The peak in the observed projected separation distribution also decreases with mass, going from 30 AU for G dwarfs (Duquennoy \& Mayor 1991), 4-30 AU for M dwarfs (Raghavan et al. 2010; Fischer \& Marcy 1992) to 6-8 AU for VLM stars and brown dwarfs (Allen 2007; Burgasser et al. 2007; Kraus \& Hillenbrand 2012).

The observed peak in the projected separation distribution for VLM dwarfs is largely based on direct imaging studies, which have discovered $>80 \%$ of the VLM binary systems to date (Burgasser et al. 2007; Bardalez Gagliuffi et al. 2014 hereafter, BG14). Angular resolution limits impose a bias on the separations observed. For ground-based telescopes with Adaptive Optics (AO) and the Hubble Space Telescope (HST), this resolution limit is roughly 0 " $05-0$ " 1 , which at the typical distances of known VLM dwarfs, $20-40 \mathrm{pc}^{5}$, corresponds to the observed peak in the projected separation distribution. Tighter systems are unresolvable. Measurements of radial velocity (RV) and astrometric variability more adequately probe the small projected separation regime, but such measurements are resource-intensive and introduce their own set of geometric biases. An alternative approach to identifying closely separated VLM binaries is as spectral binary systems.

\footnotetext{
* Some of the data presented herein were obtained at the W.M. Keck Observatory, which is operated as a scientific partnership among the California Institute of Technology, the University of California, and the National Aeronautics and Space Administration. The Observatory was made possible by the generous financial support of the W.M. Keck Foundation.

${ }_{5}^{4}$ IPAC Visiting Graduate Student Fellow 2014.

5 In order to achieve $\mathrm{S} / \mathrm{N} \gtrsim 25$ with low and high resolution spectroscopy.
}

Spectral binaries exhibit peculiarities in blended-light spectra that arise from the superposition of two components with distinct spectral morphologies. This method has been used to disentangle the spectra of white dwarf/M dwarf binaries (Silvestri et al. 2007) and more recently, VLM stars and brown dwarfs, especially those with a $\mathrm{T}$ dwarf component (e.g., Cruz et al. 2004; Metchev et al. 2008; Burgasser et al. 2010 (hereafter: B10); Geißler et al. 2011; Day-Jones et al. 2013; BG14). The identification of spectral binaries is independent of their projected separation, allowing the identification of binaries with very tight separations. The selection biases for this method (small separation, distinct component masses) are different from those of direct imaging, $\mathrm{RV}$ and astrometric variability, and overluminosity, providing a complementary approach to finding VLM binary systems.

While many brown dwarf spectral binaries have been discovered serendipitously (Cruz et al. 2004; Burgasser 2007b; Gelino \& Burgasser 2010), recent systematic searches (B10, BG14) have increased the number of known spectral binaries to $\sim 50$. Follow-up of candidates is necessary to confirm their binary nature since the spectral peculiarities that signal binarity may instead be the result of atmospheric variability, as in the case of the T1.5 2MASS J21392676+0220226 ${ }^{6}$ (Radigan et al. 2012; Khandrika et al. 2013). Only 12 spectral binaries have been confirmed by direct imaging, $\mathrm{RV}$, astrometric variability or overluminosity (See Table 8; Burgasser et al. 2006b, 2011b, 2012; Blake et al. 2008; Gelino \& Burgasser 2010; Stumpf et al. 2011; Dupuy \& Liu 2012; Faherty et al. 2012; Manjavacas et al. 2013) and many of these have turned out to be close separation systems. The M9 dwarf SDSS J0006-0852AB (Burgasser et al. 2012) and the M8.5 dwarf 2MASS J0320-0446AB (Blake et al. 2008; Burgasser et al. 2008) were confirmed as binaries by RV variability and found to have projected separations $<1$ AU. The L4 dwarf SDSS J0805+4812

\footnotetext{
6 Hereafter targets observed in this study are referred to by shorthand notation: Jhhmm $+d d m m$, where $h$ is hour, $d$ is degree and $m$ is minute. Full coordinates are listed in Table 1.
} 
(Burgasser 2007b), confirmed as a binary through astrometric variability, has a semimajor axis 0.9-2.3 AU (Dupuy \& Liu 2012). Even with the high resolution images provided by the Keck II Laser Guide Star Adaptive Optics (LGS-AO) system, none of these binaries can be resolved.

Nevertheless, high resolution imaging remains an efficient first test for binarity. In this article, we present high resolution LGS-AO observations of 43 late-M, L and T dwarfs, 17 of which are spectral binary candidates. Section 2 describes the target selection and observation procedures using the LGS-AO system and Keck II/NIRC2 (van Dam et al. 2006; Wizinowich et al. 2006). For the unresolved spectral binaries (visual and index-selected) we determine detection and separation limits in Section 3.1. We discuss in detail each of the known, unresolved binaries in Section 3.2. We report three resolved sources and describe their properties in Section 3.3. In Section 4.1 we analyze multi-epoch AO images of SDSS J2052-1609 and determine a first astrometric orbit for this $\mathrm{L} / \mathrm{T}$ transition system. For the other two resolved systems, we estimate orbital parameters with Monte Carlo methods in Section 4.2. We discuss the broader implications of our results in the context of small separation VLM binaries in Section 4.3. Our results are summarized in Section 5.

\section{TARGET SELECTION AND OBSERVATIONS}

\subsection{Spectral Binary Identification}

The 43 sources observed in our study (Table 1) were selected from known late-M, L and T dwarfs in the vicinity of the Sun with a suitable tip-tilt star for LGS-AO correction. These include 33 M9-T3 dwarfs initially classified as spectral binaries by visual inspection, before the $\mathrm{B} 10$ and BG14 selection criteria had been defined. We re-examined their binary candidacy by dividing them into two groups according to spectral type: 15 objects in the M9-L7 range analyzed with the BG14 method, and 22 objects in the L5-T3 range, analyzed with the B10 method. The four objects overlapping in these spectral type ranges were analyzed by both methods. Ten other low mass stars and brown dwarfs were also observed as backup targets, but were excluded from the analysis because visual inspection rejected them as spectral binary candidates.

Indices were measured from low resolution $(\lambda /$ $\Delta \lambda=75-120$ ), near-infrared IRTF/SpeX spectra (Rayner et al. 2003) covering $0.9-2.4 \mu \mathrm{m}$, accessed from the SpeX Prism Libraries (Burgasser 2014). One of our targets, 2MASS $\mathrm{J} 2126+7617$, has a declination outside the observable range of SpeX/IRTF $\left(-50^{\circ}<\delta<+67^{\circ}\right)$, so a smoothed Keck/ NIRSPEC spectrum was used instead (Kirkpatrick et al. 2010). Spectral indices given in B10 and BG14 were calculated for each spectrum, and regions of interest (ROI) in index-index spaces were delineated using confirmed binaries. Slight modifications to the limits of some ROIs in both B10 and BG14 were made to include known binaries WISEP J0720-0846 and 2MASS J1209-1004 (Burgasser et al. 2015a; Liu et al. 2010, respectively), which had not been detected at the time the index selection criteria were defined. Table 2 shows the updated limits of the index selection ROIs for both sets of criteria. Strong and weak candidates are selected by the number of times they fall within the ROIs, as described in B10 and BG14.

From the BG14 set, 8 sources were selected as candidates from spectral indices (4 as strong, 4 as weak). Single and binary templates were fit to these index-selected sources, ranked by a $\chi^{2}$ statistic. The best fit single and binary templates were compared to each other with an F-test to assess the percentage confidence that the binary fit is statistically better than the single fit. The primary types were constrained to \pm 3 subtypes from the combined optical spectral type or, in its absence, near infrared type, and the secondary types were allowed to vary between T1 and T8. After template fitting, 6 sources remained as candidates. From the B10 set, 16 sources were selected as index candidates (11 as strong, 5 as weak) and after fitting, 12 sources remained as candidates. 2MASS J1711 +2232 was selected as a candidate on both sets. In all, we classify 17 sources as true spectral binary candidates (Table 1), close to half of the visually selected spectral binaries.

\subsection{NIRC2 High Resolution Imaging and Reduction}

High angular resolution images of our targets were obtained using the Keck II LGS-AO system with NIRC2 on nine nights between 2009 August and 2014 January. Tip-tilt reference stars within $60^{\prime \prime}$ of the targets were selected from the USNO-B catalog (Monet et al. 2003). A 3-point dither pattern was used to avoid the noisy lower left quadrant of the array, and was repeated as needed with different dither offsets to build up long exposures. Total integration times were between 60 and $720 \mathrm{~s}$, depending on the brightness of the source and the atmospheric conditions. All objects were observed with the Mauna Kea Observatories (MKO) $H$ filter (Simons \& Tokunaga 2002; Tokunaga et al. 2002) and narrow plate scale (9.970 \pm $0.012 \mathrm{mas} / \mathrm{pixel}$ for a single-frame field of view of $10^{\prime \prime} \times 10^{\prime \prime}$; Pravdo et al. 2006). The MKO $J$ and/or $K_{s}$ filters were also used for targets with apparent companions.

The images were reduced in a standard fashion using interactive data language (IDL) scripts. First, a dark frame was subtracted from each science frame. For each science exposure a sky frame was constructed from the median average of all images acquired for the target, exclusive of the frame being reduced. The sky-subtracted frames were then divided by a normalized dome flat. A bad pixel mask was applied to smooth over bad pixels using the average of the neighboring pixels. All images in a given epoch and common filter were shifted to align the target to a common location, and the stack was median-combined to create the final mosaics.

\section{ANALYSIS}

\subsection{Image Characterization and Companion Detection Limits for Unresolved Sources}

The reduced image mosaics around each target are shown in Figures 1 and 2. Strehl and signal-to-noise ratios $(\mathrm{S} / \mathrm{N})$ are reported in Table 1 . The Strehl ratio was calculated by comparing each point source to a theoretical, diffractionlimited, monochromatic, NIRC2 point-spread function (PSF) with the NIRC2Strehl IDL routine. ${ }^{7}$ The $\mathrm{S} / \mathrm{N}$ was computed assuming Poisson statistics:

$$
\mathrm{S} / \mathrm{N}=\frac{N_{\text {star }}}{\sqrt{n_{\text {sky }} \sigma_{\text {sky }}^{2}+\frac{N_{\text {star }}}{g}}}
$$

\footnotetext{
Retrieved from https://www2.keck.hawaii.edu/optics/lgsao/software/
} 
Table 1

Observation Log

\begin{tabular}{|c|c|c|c|c|c|c|c|c|c|}
\hline Name & SpT & 2MASS $H$ & Date & Reference Star & Filter & $t_{\exp }(\mathrm{s})$ & Airmass & Strehl Ratio & $\mathrm{S} / \mathrm{N}$ \\
\hline \multicolumn{10}{|c|}{ Spectral Binaries from B10 and BG14 } \\
\hline \multirow[t]{3}{*}{ SDSS J011912.22+240331.6 } & $\mathrm{T} 2$ & $16.46 \pm 0.03$ & 2009 Aug 15 & $1140-0016097$ & $H$ & 720 & 1.02 & 0.25 & 717 \\
\hline & & & 2013 Sep 22 & $1140-0016097$ & $H$ & 120 & 1.01 & 0.01 & 103 \\
\hline & & & 2013 Sep 23 & $1140-0016097$ & $H$ & 120 & 1.05 & 0.23 & 897 \\
\hline SDSS J024749.90-163112.6 & $\mathrm{T} 2$ & $16.31 \pm 0.03$ & 2009 Aug 15 & 0734-0037544 & $H$ & 720 & 1.32 & 0.11 & 764 \\
\hline 2MASS J03440892+0111251 & L0.5 & $13.91 \pm 0.04$ & 2013 Sep 22 & $0911-0037820$ & $H$ & 175 & 1.07 & 0.15 & 3043 \\
\hline SDSS J035104.37+481046.8 & $\mathrm{T} 1$ & $15.57 \pm 0.14$ & 2009 Aug 15 & $1381-0118655$ & $H$ & 720 & 1.38 & 0.14 & 894 \\
\hline 2MASS J05185995-2828372 & $\mathrm{T} 1$ & $14.83 \pm 0.07$ & 2013 Sep 23 & $0615-0055796$ & $H$ & 120 & 1.63 & 0.12 & 1718 \\
\hline WISE J07200320-0846513 & M9.5 & $9.92 \pm 0.02$ & 2014 Jan 19 & $0812-0137371$ & $H$ & 60 & 1.28 & 0.02 & 1638 \\
\hline \multirow[t]{2}{*}{ SDSS J080531.84+481233.0 } & L9 & $13.92 \pm 0.04$ & 2010 Mar 24 & $1382-0223846$ & $K_{s}$ & 540 & 1.21 & 0.12 & 2624 \\
\hline & & & 2013 Sep 23 & $1382-0223846$ & $H$ & 120 & 1.61 & 0.09 & 3029 \\
\hline 2MASS J11061197+2754225 & $\mathrm{T} 2.5$ & $14.15 \pm 0.05$ & 2010 Mar 24 & 1179-0233699 & $H$ & 720 & 1.03 & 0.07 & 2559 \\
\hline 2MASS J12095613-1004008 & $\mathrm{T} 3$ & $15.33 \pm 0.09$ & 2014 Jan 13 & 0799-0230529 & $H$ & 120 & 1.15 & 0.04 & 1146 \\
\hline \multirow[t]{4}{*}{ 2MASS J13411160-3052505 } & L3 & $13.72 \pm 0.03$ & 2014 Jan 13 & 0591-0304901 & $J$ & 120 & 1.61 & 0.02 & 807 \\
\hline & & & 2014 Jan 13 & 0591-0304901 & $H$ & 120 & 1.65 & 0.04 & 1508 \\
\hline & & & 2014 Jan 19 & 0591-0304901 & $H$ & 120 & 1.59 & 0.04 & 1845 \\
\hline & & & 2014 Jan 13 & 0591-0304901 & $K_{s}$ & 180 & 1.59 & 0.05 & 1861 \\
\hline \multirow[t]{2}{*}{ SDSS J143553.25+112948.6 } & $\mathrm{T} 2$ & $16.52 \pm 0.04$ & 2009 Aug 15 & $1014-0229971$ & $H$ & 360 & 1.25 & 0.05 & 373 \\
\hline & & & 2009 Aug 15 & $1014-0229971$ & $K_{s}$ & 360 & 1.30 & 0.08 & 268 \\
\hline \multirow[t]{4}{*}{ SDSS J151114.66+060742.9 } & T0 & $14.96 \pm 0.08$ & 2009 Aug 15 & $0961-0243717$ & $J$ & 360 & 1.36 & 0.01 & 560 \\
\hline & & & 2009 Aug 15 & 0961-0243717 & $H$ & 360 & 1.27 & 0.09 & 1078 \\
\hline & & & 2009 Aug 15 & $0961-0243717$ & $K_{s}$ & 720 & 1.32 & 0.13 & 1101 \\
\hline & & & 2010 May 19 & 0961-0243717 & $H$ & 120 & 1.03 & 0.09 & 94 \\
\hline SDSS J151643.01+305344.4 & T0.5 & $15.87 \pm 0.16$ & 2010 Mar 24 & 0930-0297471 & $H$ & 720 & 1.04 & 0.06 & 1007 \\
\hline SDSS J154727.23+033636.3 & L2 & $15.07 \pm 0.06$ & 2009 Aug 15 & 0936-0258682 & $H$ & 720 & 1.40 & 0.01 & 805 \\
\hline \multirow[t]{2}{*}{ 2MASS J1711457+223204 } & L6.5 & $15.80 \pm 0.11$ & 2010 May 13 & $1125-0317350$ & $J$ & 720 & 1.22 & 0.05 & 381 \\
\hline & & & 2010 May 13 & $1125-0317350$ & $H$ & 720 & 1.27 & 0.07 & 844 \\
\hline \multirow[t]{2}{*}{ 2MASS J1733423-165449 } & L0.5 & $12.81 \pm 0.06$ & 2009 Aug 15 & 0730-0518366 & $J$ & 720 & 1.39 & 0.08 & 2324 \\
\hline & & & 2009 Aug 15 & $0730-0518366$ & $H$ & 720 & 1.36 & 0.10 & 2748 \\
\hline \multirow[t]{3}{*}{ SDSS J205235.31-160929.8 } & $\mathrm{T} 1$ & $15.45 \pm 0.03$ & 2009 Aug 15 & $0738-0802833$ & $J$ & 360 & 1.30 & 0.06 & 543 \\
\hline & & & 2009 Aug 15 & $0738-0802833$ & $H$ & 360 & 1.27 & 0.00 & 1003 \\
\hline & & & 2009 Aug 15 & $0738-0802833$ & $K_{s}$ & 360 & 1.28 & 0.17 & 729 \\
\hline
\end{tabular}

Visually Selected Spectral Binary Candidates

\begin{tabular}{|c|c|c|c|c|c|c|c|c|c|}
\hline \multirow{2}{*}{ 2MASS J0019457+521317 } & M9 & $12.07 \pm 0.02$ & 2009 Aug 15 & $1422-0011510$ & $J$ & 360 & 1.30 & 0.09 & 2502 \\
\hline & & & 2009 Aug 15 & $1422-0011510$ & $H$ & 720 & 1.28 & 0.25 & 5028 \\
\hline 2MASS J00320509+0219017 & L1.5 & $13.39 \pm 0.02$ & 2009 Aug 15 & 0923-0006944 & $H$ & 720 & 1.10 & 0.11 & 2811 \\
\hline \multirow{2}{*}{ SDSS J003259.36+141036.6 } & L8 & $15.65 \pm 0.14$ & 2009 Aug 15 & $1041-0005438$ & $H$ & 720 & 1.02 & 0.10 & 1112 \\
\hline & & & 2009 Aug 15 & $1041-0005438$ & $K_{s}$ & 360 & 1.02 & 0.11 & 721 \\
\hline 2MASS J02361794+0048548 & L6.5 & $15.27 \pm 0.07$ & 2013 Sep 22 & 0908-0027044 & $H$ & 180 & 1.06 & 0.14 & 1959 \\
\hline SDSS J075840.33+324723.4 & $\mathrm{T} 2$ & $14.11 \pm 0.04$ & 2010 Mar 24 & $1227-0198441$ & $H$ & 720 & 1.06 & 0.05 & 2333 \\
\hline SDSS J093109.56+032732.5 & L7.5 & $16.27 \pm 0.24$ & 2010 Mar 24 & 0934-0195871 & $H$ & 720 & 1.18 & 0.03 & 611 \\
\hline 2MASS J09490860-1545485 & $\mathrm{T} 2$ & $15.26 \pm 0.11$ & 2010 Mar 24 & 0742-0216967 & $H$ & 720 & 1.24 & 0.05 & 1399 \\
\hline SDSS J103321.92+400549.5 & L6 & $16.05 \pm 0.04$ & 2010 Mar 24 & $1300-0206652$ & $H$ & 720 & 1.07 & 0.06 & 828 \\
\hline SDSS J112118.57+433246.5 & L7.5 & $16.56 \pm 0.04$ & 2010 Mar 24 & $3015-00408-1$ & $H$ & 720 & 1.16 & 0.08 & 674 \\
\hline \multirow[t]{2}{*}{ 2MASS J11582073+0435022 } & sdL7 & $14.68 \pm 0.06$ & 2010 May 13 & 0945-0201532 & $H$ & 720 & 1.04 & 0.15 & 1776 \\
\hline & & & 2010 May 13 & 0945-0201532 & $K_{s}$ & 720 & 1.04 & 0.23 & 1882 \\
\hline SDSS J120602.51+281328.7 & $\mathrm{T} 3$ & $15.83 \pm 0.03$ & 2010 Mar 24 & $1182-0220446$ & $H$ & 720 & 1.11 & 0.10 & 197 \\
\hline 2MASS J14283132+5923354 & L4 & $13.88 \pm 0.04$ & 2010 May 19 & $1493-0219430$ & $H$ & 720 & 1.29 & 0.10 & 3219 \\
\hline 2MASS J1707333+430130 & L0.5 & $13.18 \pm 0.03$ & 2009 Aug 15 & $1330-0334426$ & $H$ & 720 & 1.41 & 0.08 & 2425 \\
\hline \multirow[t]{2}{*}{ 2MASS J1721039+334415 } & L3 & $12.95 \pm 0.03$ & 2009 Aug 15 & $1237-0272128$ & $H$ & 720 & 1.21 & 0.11 & 953 \\
\hline & & & 2010 Mar 24 & $1237-0272128$ & $H$ & 720 & 1.03 & 0.07 & 2721 \\
\hline 2MASS J21265916+7617440 & T0 & $13.59 \pm 0.04$ & 2013 Sep 23 & $1662-0097897$ & $H$ & 120 & 2.16 & 0.08 & 79 \\
\hline SDSS J214956.55+060334 & M9 & $12.63 \pm 0.03$ & 2009 Aug 15 & 0960-0568745 & $H$ & 720 & 1.08 & 0.09 & 2670 \\
\hline \multicolumn{10}{|c|}{ Additional Targets } \\
\hline WISEP J004701.06+680352.1 & L7.5 & $13.97 \pm 0.04$ & 2013 Sep 23 & $1580-0021858$ & $H$ & 90 & 1.56 & 0.28 & 2410 \\
\hline 2MASS J03001631+2130205 & L6p & $14.73 \pm 0.07$ & 2013 Sep 22 & $1115-0038422$ & $H$ & 120 & 1.06 & 0.13 & 1583 \\
\hline 2MASS J03020122+1358142 & L3 & $15.43 \pm 0.09$ & 2013 Sep 23 & $1039-0030470$ & $H$ & 120 & 1.01 & 0.05 & 944 \\
\hline \multirow[t]{2}{*}{ НYT0429+1535 } & $\ldots$ & $\ldots$ & 2013 Sep 22 & $1031-0059287$ & $\mathrm{CH}_{4}$ & 120 & 1.01 & 0.16 & 131 \\
\hline & & & 2013 Sep 22 & $1031-0059287$ & $H$ & 120 & 1.00 & 0.17 & 88 \\
\hline 2MASSI J0443058-320209 & L5 & $14.35 \pm 0.06$ & 2013 Sep 22 & 0579-0075735 & $H$ & 120 & 1.67 & 0.07 & 2206 \\
\hline
\end{tabular}


Table 1

(Continued)

\begin{tabular}{|c|c|c|c|c|c|c|c|c|c|}
\hline Name & SpT & 2MASS $H$ & Date & Reference Star & Filter & $t_{\mathrm{exp}}(\mathrm{s})$ & Airmass & Strehl Ratio & $\mathrm{S} / \mathrm{N}$ \\
\hline WISE J052857.69+090104.4 & M9.5p & $15.44 \pm 0.12$ & 2014 Jan 19 & 0990-0058827 & $H$ & 135 & 1.40 & 0.07 & 99 \\
\hline SDSS J115013.17+052012.3 & L6 & $15.46 \pm 0.14$ & 2014 Jan 13 & 0279-01016-1 & $H$ & 60 & 1.03 & 0.17 & 993 \\
\hline ULAS J132605.18+120009.9 & T6p & $17.93 \pm 0.09$ & 2010 May 19 & $1019-0249297$ & $H$ & 720 & 1.02 & 0.24 & 371 \\
\hline 2MASS J14140586+0107102 & L4.8: & $15.73 \pm 0.19$ & 2010 May 13 & 0317-00292-1 & $H$ & 720 & 1.07 & 0.20 & 1168 \\
\hline 2MASS J20025073-0521524 & L6 & $14.28 \pm 0.05$ & 2009 Aug 15 & 0846-0581639 & $H$ & 360 & 1.35 & 0.12 & 1709 \\
\hline
\end{tabular}

Table 2

Updated Index Selection Criteria for B10 and BG14

\begin{tabular}{|c|c|c|}
\hline$x$ & $y$ & Limits \\
\hline \multicolumn{3}{|r|}{ Burgasser et al. (2010) } \\
\hline $\mathrm{H}_{2} \mathrm{O}-\mathrm{J}$ & $\mathrm{H}_{2} \mathrm{O}-\mathrm{K}$ & $0.325<x<0.65$ and $y>0.615 x+0.300$ \\
\hline $\mathrm{CH}_{4}-\mathrm{H}$ & $\mathrm{CH}_{4}-\mathrm{K}$ & $0.6<x<1.0$ and $y>1.063 x-0.288$ \\
\hline $\mathrm{CH}_{4}-\mathrm{H}$ & $K / J$ & $0.65<x<1.00$ and $y>0.471 x-0.096$ \\
\hline $\mathrm{H}_{2} \mathrm{O}-\mathrm{H}$ & $H$-dip & $0.44<x<0.68$ and $y<0.49$ \\
\hline $\mathrm{SpT}$ & $\begin{array}{l}\mathrm{H}_{2} \mathrm{O}-\mathrm{J} / \\
\quad \mathrm{H}_{2} \mathrm{O}-\mathrm{H}\end{array}$ & $\begin{array}{l}\text { L8.5 }<x<\mathrm{T} 3.5, y<0.925 \text { and } y<-0.037 \mathrm{x} \\
\quad+2.106\end{array}$ \\
\hline $\mathrm{SpT}$ & $\begin{array}{l}\mathrm{H}_{2} \mathrm{O}-\mathrm{J} / \\
\quad \mathrm{CH}_{4}-K\end{array}$ & $\mathrm{~L} 8<x<\mathrm{T} 4.5$ and $y<0.041 \mathrm{x}-0.517$ \\
\hline \multicolumn{3}{|r|}{ Bardalez Gagliuffi et al. (2014) } \\
\hline SpT & $\mathrm{CH}_{4}-H$ & $\begin{array}{l}M 7.5<x<L 8 \text { and } \\
\quad y<-4.3 \times 10^{-4} x^{2}+0.0253 x+0.6824\end{array}$ \\
\hline $\mathrm{H}_{2} \mathrm{O}-\mathrm{J}$ & $\mathrm{CH}_{4}-H$ & $0.60<x<0.92$ and $y<-0.094 x+1.096$ \\
\hline $\mathrm{H}_{2} \mathrm{O}-\mathrm{J}$ & $H$-bump & $0.65<x<0.90$ and $y>0.16 x+0.806$ \\
\hline $\mathrm{CH}_{4}-\mathrm{J}$ & $\mathrm{CH}_{4}-\mathrm{H}$ & $0.6<x<1.04, y<1.04$ and $y<-0.562 x+1.417$ \\
\hline $\mathrm{CH}_{4}-J$ & $H$-bump & $0.60<x<0.74, y>0.91$ and $y>1.00 x+0.24$ \\
\hline $\mathrm{CH}_{4}-\mathrm{H}$ & $J$-slope & $0.94<x<1.03, y>1.03$ and $y>1.250 \mathrm{x}-0.207$ \\
\hline $\mathrm{CH}_{4}-\mathrm{H}$ & $J$-curve & $0.95<x<1.03$ and $y>1.245 x^{2}-1.565 x+2.312$ \\
\hline $\mathrm{CH}_{4}-\mathrm{H}$ & $H$-bump & $\begin{aligned} 0.94 & <x<1.04, y>0.92 \text { and } \\
y & <1.36 x^{2}-4.26 x+3.877\end{aligned}$ \\
\hline$J$-slope & $H$-dip & $1.03<x<1.13$ and $y<0.20 \mathrm{x}+0.27$ \\
\hline$J$-slope & $H$-bump & $1.025<x<1.130, y>-2.75 x+3.84$ and $y>0.91$ \\
\hline$K$-slope & $\mathrm{H}_{2} \mathrm{O}-Y$ & $\begin{array}{l}0.93<x<0.96 \text { and } \\
y>12.036 x^{2}-20.000 x+9.037\end{array}$ \\
\hline$J$-curve & $H$-bump & $\begin{array}{l}2.00<x<2.45, y>0.92 \text { and } \\
\quad y>0.269 x^{2}-1.326 x+2.527\end{array}$ \\
\hline
\end{tabular}

where $N_{\text {star }}$ is the total counts from the star at a radius of 1.5 times the full width at half maximum, $n_{\text {sky }}$ is the number of pixels used for the standard deviation of the sky counts, $\sigma_{\text {sky, }}$, which encompasses noise from several sources (read out, dark current, image reduction, etc.) and $g$ is the gain in $\mathrm{DN} / e^{-}$(data number per electron).

Three of our sources are resolved: 2MASS 1341-3052, SDSS 1511+0607 and SDSS 2052-1609; these are shown in Figure 1 and discussed further in Section 3.3. One source, 2MASS J1733-1654, has a feature that we cannot distinguish between bona fide source and PSF structure, so we consider this to be a "source of interest." The remaining sources are unresolved at the limits of our sensitivity and image quality. Because the PSF of the images vary considerably, we determined detection limits through a source implantation simulation of representative images. We organized the targets by Strehl and $\mathrm{S} / \mathrm{N}$ and selected two representative sources of high Strehl (WISE J0047+6308) and low Strehl (2MASS
J0032+0219), as shown in Figure 3. For these sources, we simulated binary companions by scaling down the brightness of each image, and then shifting and superimposing it onto the original image. The implanted image was scaled down by a maximum of $6 \mathrm{mag}$, which was the largest magnitude difference inferred from the template fitting of spectral binary candidates, and shifted by up to 50 pixels or $\sim 0$." 5 in any angle. Magnitude difference, separation and position angle were all drawn from a uniform random distribution.

We visually examined each image at multiple contrast ratios to search for the implanted companion. This experiment was performed $N \gtrsim 12,000$ times per source, varying the target, scale factor and offset. A "detection" required clicking within 15 pixels of the implanted secondary, with the option to decide if an implanted companion was visually undetectable. We determined the maximum relative magnitude as a function of separation for which the detection fraction exceeded $50 \%$. The detection fraction was calculated in steps of $0.5 \mathrm{mag}$ and $0 . " 05$, sliding by half a step along both axes for a total of $\sim 400$ overlapping bins. Figure 4 shows that the PSF dominates the sensitivity close to the star centroid. For the case of low Strehl ratio, detections reach a minimum at $\Delta H \approx 5$ mag, 0 " 3 away from the center of the PSF, beyond which our sensitivity is limited by sky noise. For the high Strehl ratio case the floor lies around $5.5 \mathrm{mag}$ difference at radii greater than 0 ." 4 .

We applied the sensitivity curves of our representative sources to systems with similar Strehl ratios. For the 30 unresolved spectral binary candidates, we compared these sensitivity limits to the magnitude differences predicted from template fitting to determine separation limits (Table 3). Figure 5 shows an example of the sensitivity curve and separation constraint for the spectral binary candidate 2MASS $\mathrm{J} 1711+2232$. Five of our unresolved spectral binaries have been previously confirmed as true binaries (See Section 3.2), but our separation limits are up to $40 \%$ greater, i.e., these binaries can not be resolved with our observations. For the case of 2MASS J1209-1004, our estimated separation limit is smaller than the measured separation, suggesting that the secondary has moved to a closer configuration. Similarly, for our three resolved systems the calculated separation limit is always smaller than the measured separation, which means that our separation limits correctly constrain the PSF of the primary. The remaining 9 unresolved spectral binaries have angular separation limits between 0 ". 04 and 0 ". 28 .

\subsection{Unresolved Known Binaries}

\subsubsection{MASS J05185995-2828372}

2MASS J0518-2828 was the first source to be identified as a spectral binary of L6 and T4 components (Cruz et al. 2004) and was marginally resolved with HST (Burgasser et al. 2006b) 


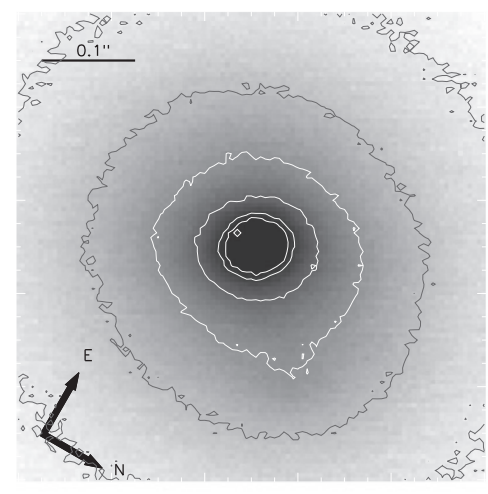

(a) 2MASS J1341-3052 J.

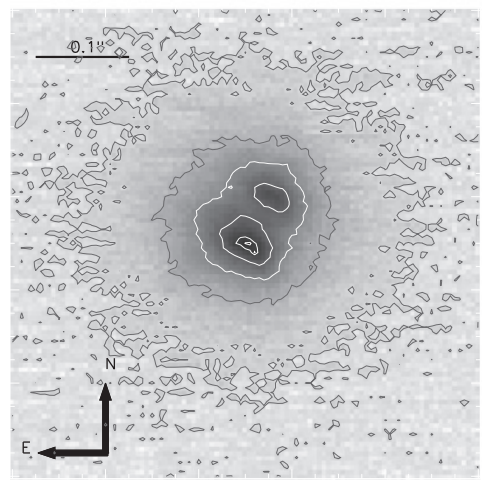

(d) SDSS J1511+0607 J.

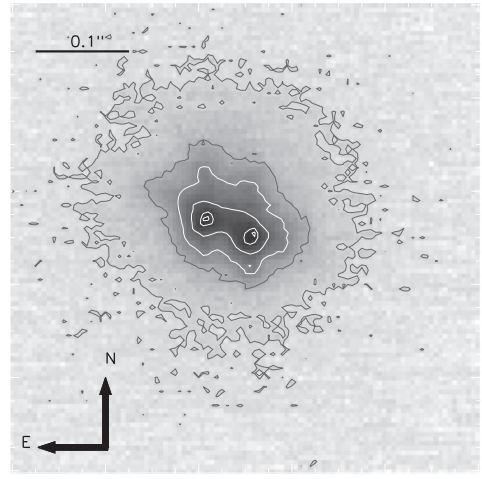

(g) SDSS J2052-1609 J.

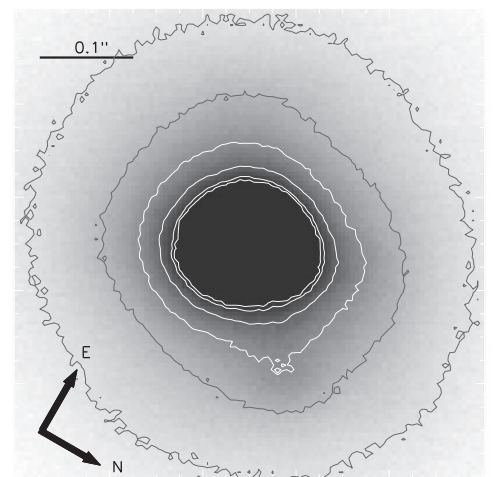

(b) 2MASS J1341-3052 H.

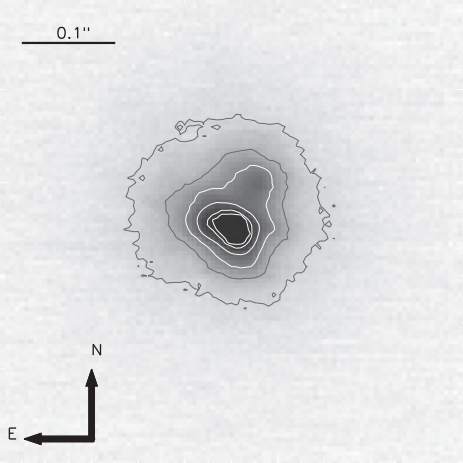

(e) SDSS J1511+0607 H.

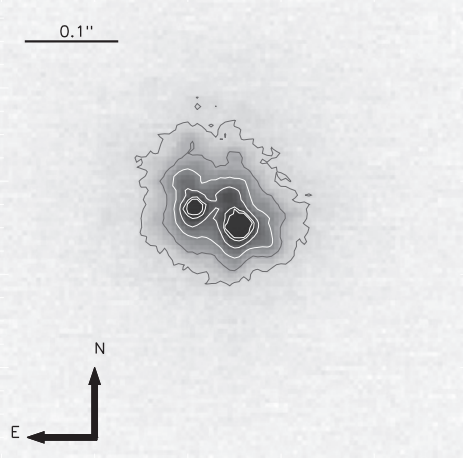

(h) SDSS J2052-1609 H.

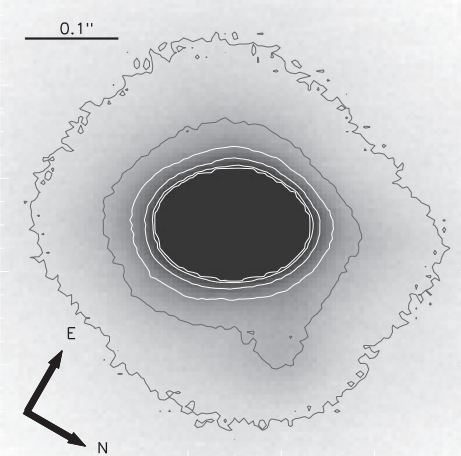

(c) 2MASS J1341-3052 $K_{s}$.

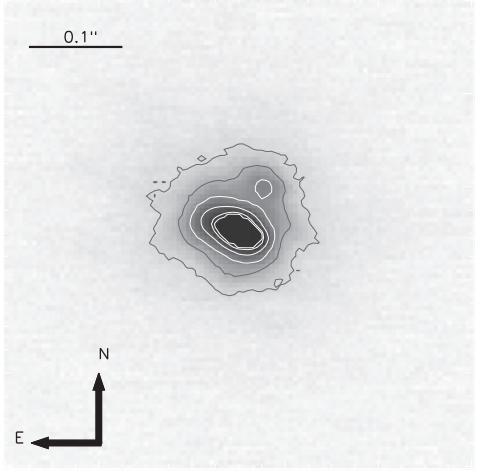

(f) SDSS J1511+0607 $K_{s}$.

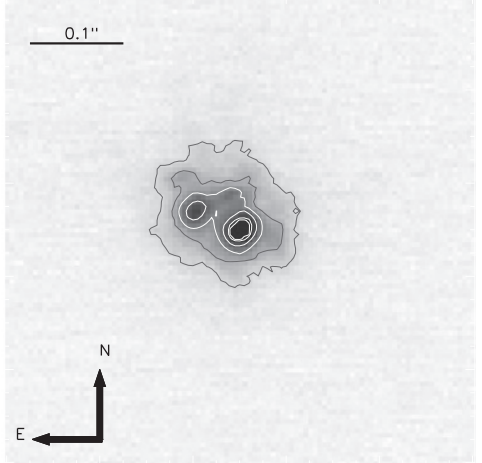

(i) SDSS J2052-1609 $\mathrm{K}_{\mathrm{s}}$.

Figure 1. Keck NIRC2 LGS-AO images of the three binaries resolved in this sample in $J H K_{s}$ bands.

with an angular separation of $0 . " 051 \pm 0$. 012. Dupuy \& Liu (2012) find a small astrometric perturbation for this source that cannot be clearly attributed to orbital motion. Konopacky et al. (2010) observed this source with LGS-AO at Keck in 2006 and were not able to resolve it. Its parallactic distance has been measured to be $22.9 \pm 0.4 \mathrm{pc}$ (Dupuy \& Liu 2012), implying a projected separation of $1.17 \pm 0.28 \mathrm{AU}$ from the $H S T$ measurement. Our LGS-AO observations also fail to resolve this system to a limit of 98 mas or $2.2 \mathrm{AU}$, which is consistent with the $H S T$ observations. This system appears to be a very tight binary whose separation is just below the limits of ground-based $\mathrm{AO}$ imaging.

\subsubsection{WISEP J072003.20-084651.2}

WISEP J0720-0846 was discovered by Scholz (2014) and confirmed by Burgasser et al. (2015a) as an M9 at a distance of $6.0 \pm 1.0 \mathrm{pc}$. The latter study identified a candidate companion 


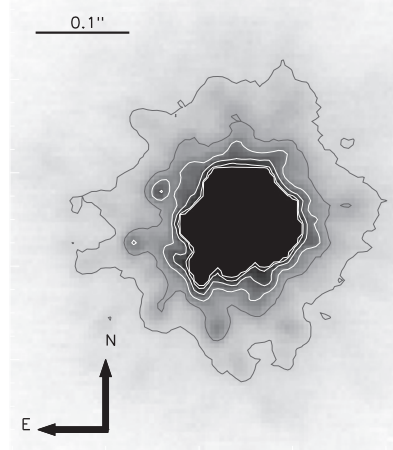

(i) $2 M A S S I J 0019+5213$.

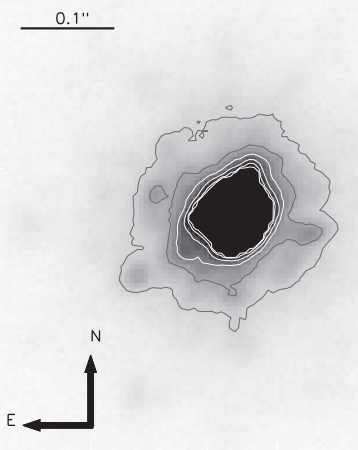

(iv) WISEP J0047+6803.

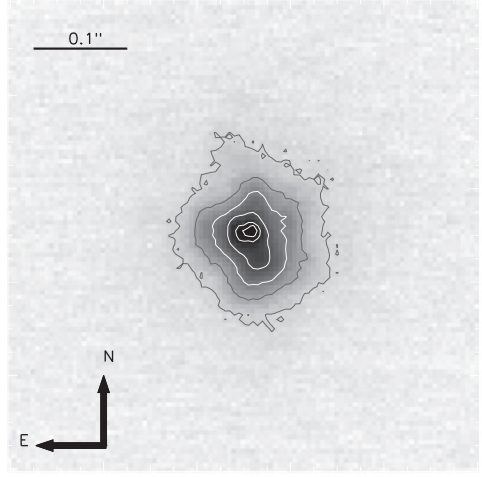

(vii) SDSS J0247-1631.

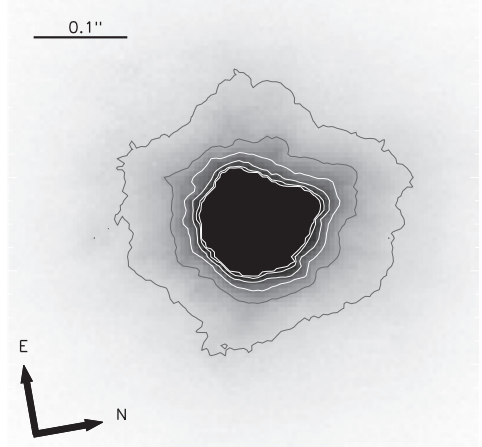

(x) $2 M A S S J 0344+0110$.

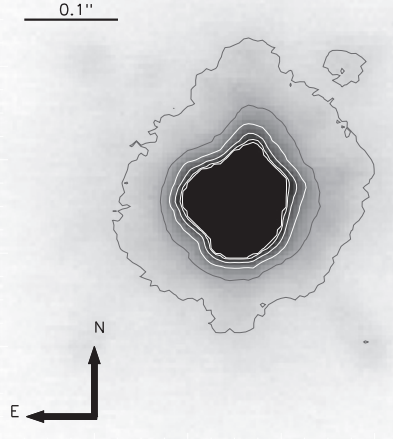

(ii) $2 M A S S J 0032+0219$.

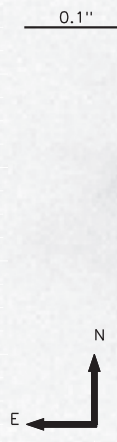

(v) SDSS J0119+2403.

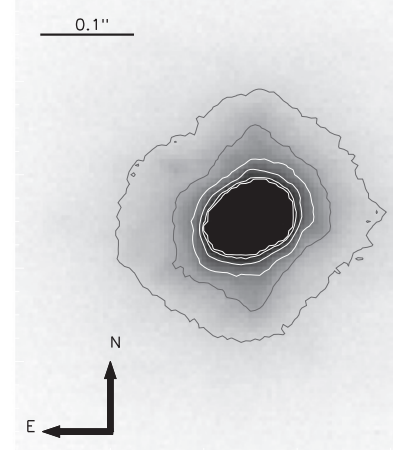

(viii) $2 M A S S J 0300+2130$.

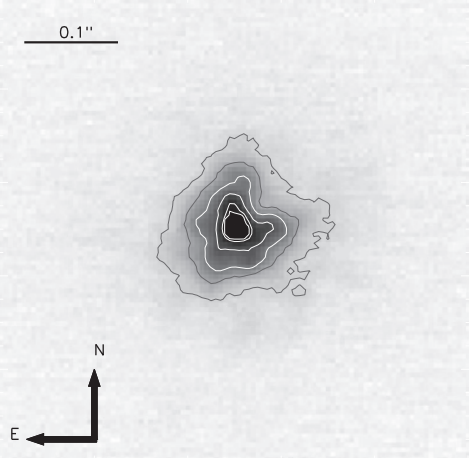

(xi) SDSS J0351+4810.

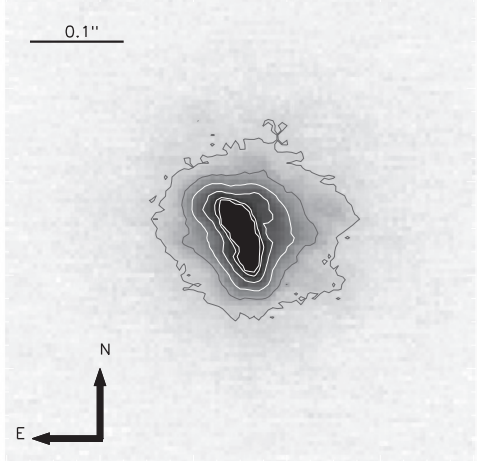

(iii) SDSS J0032+1410.
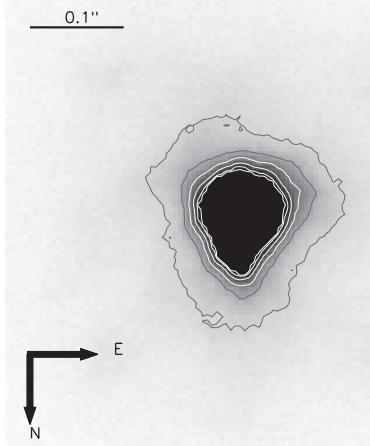

(vi) $S D S S p$ J0236+0048.

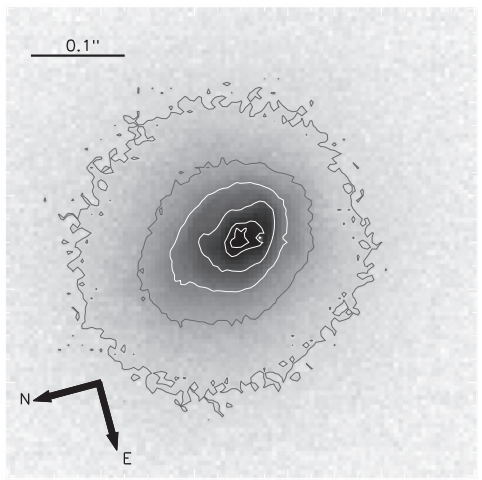

(ix) $2 M A S S$ J0302+1358.

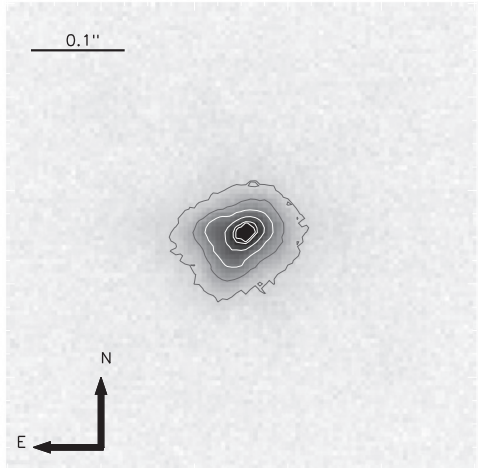

(xii) HTY J0429+1535.

Figure 2. Keck NIRC2 LGS-AO images in the $H$ band of all targets. Contours are drawn at 20, 40, 60, 80, 95 and $99 \%$ of the image minimum. 
The Astronomical Journal, 150:163 (18pp), 2015 November

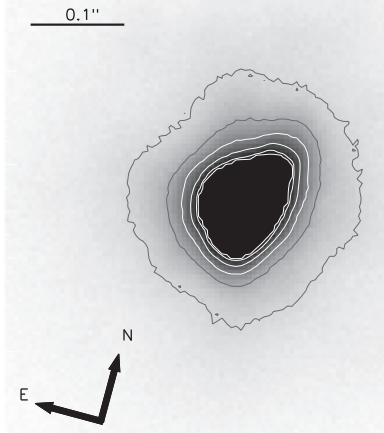

(i) 2MASS J0443-3202.

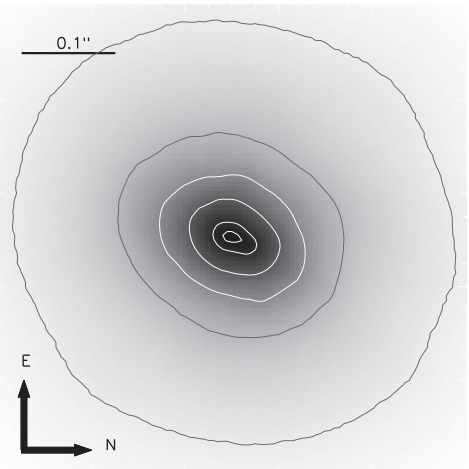

(iv) WISE J0720-0846.

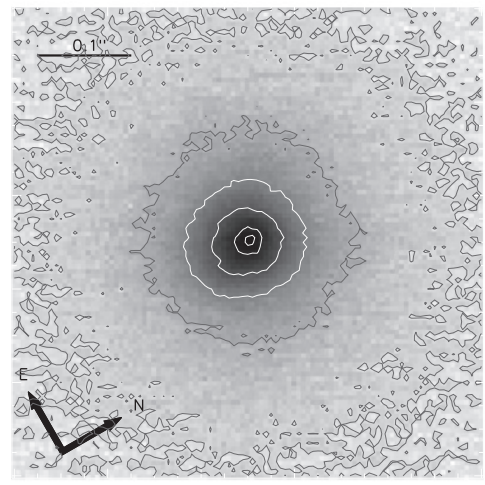

(vii) SDSS J0931+032\%.

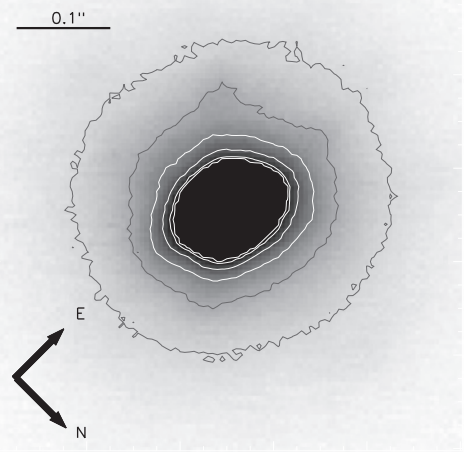

(x) $2 M A S S J 1106+2754$.

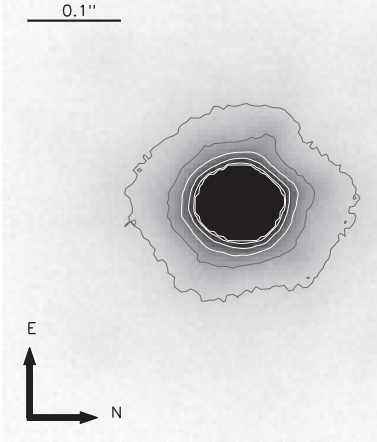

(ii) $2 M A S S J 0518-2828$.

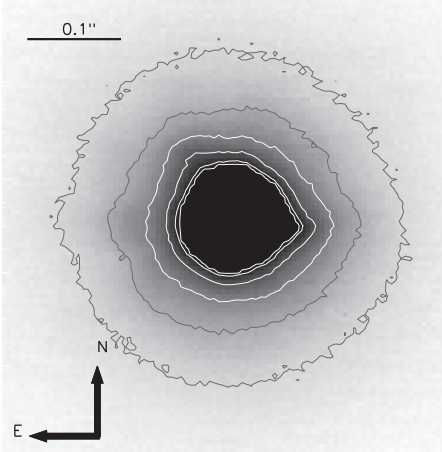

(v) SDSS J0758+3247.

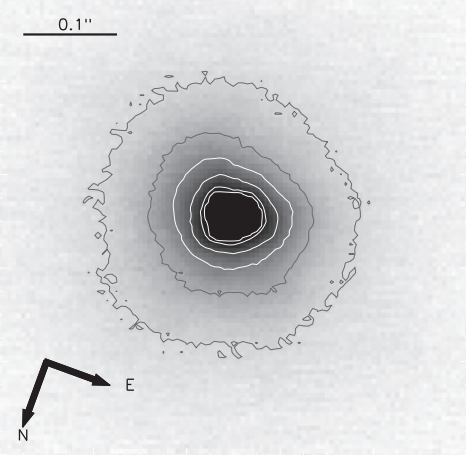

(viii) 2MASS J0949-1545.

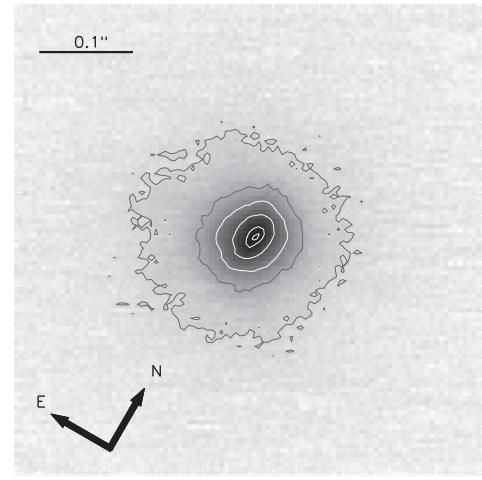

(xi) SDSS J1121+4332.

Figure 2. (Continued.)
Bardalez Gagliuffi, Gelino, \& Burgasser

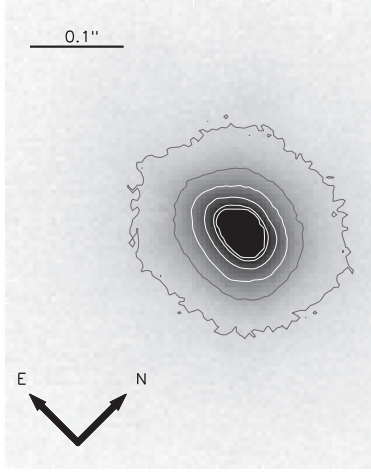

(iii) WISE J0528+0901.

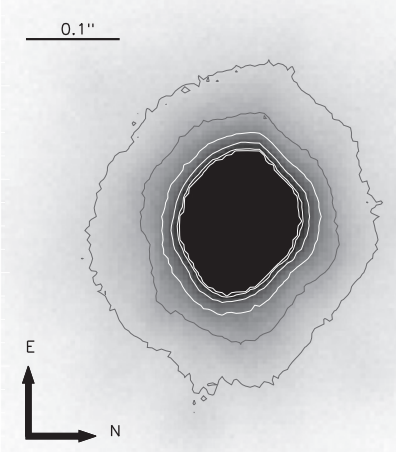

(vi) SDSS J0805+4812.

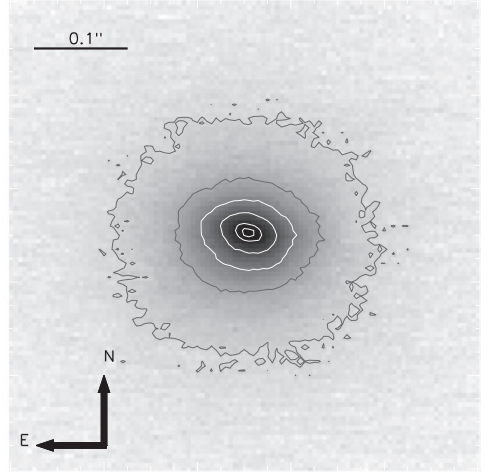

(ix) SDSS J1033+4005.

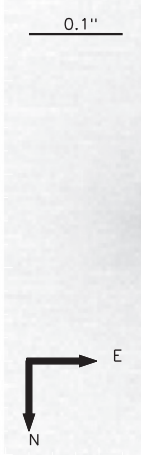

(xii) 2MASS J1150+0520. 


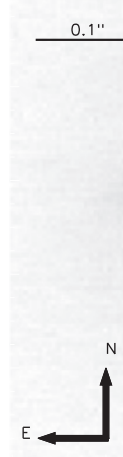

(i) $2 M A S S J 1158+0435$.

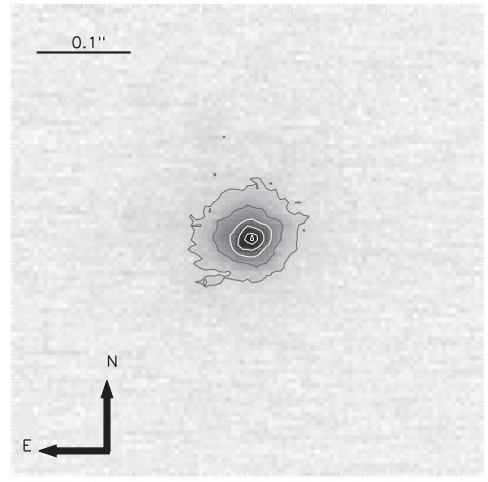

(iv) $U L A S J 1326+1200$.

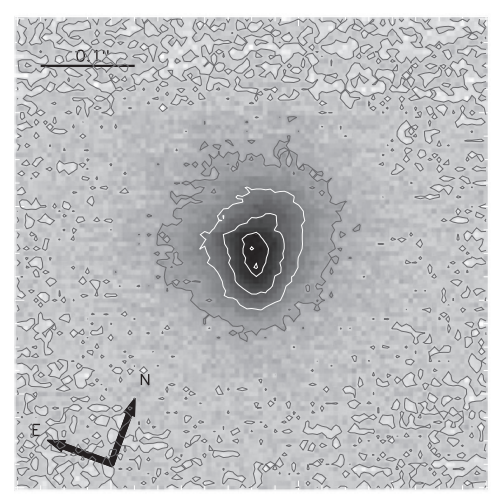

(vii) $S D S S J 1435+1129$.

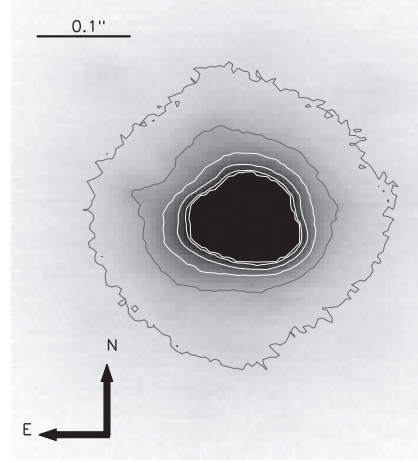

(x) $2 M A S S J 1707+4301$.

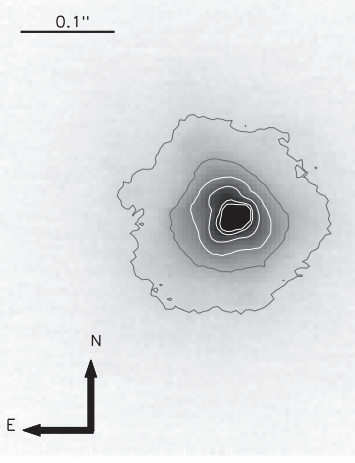

(ii) SDSS J1206+2813.

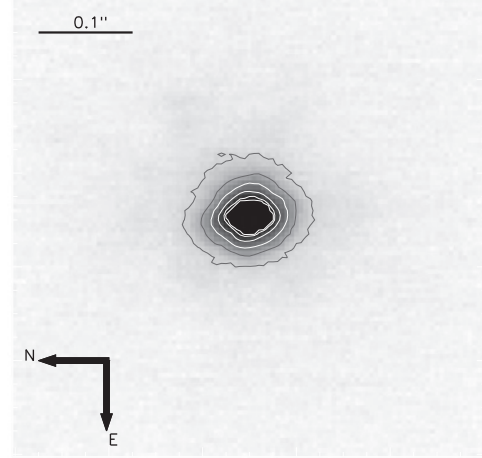

(v) $2 M A S S J 1414+0107$.

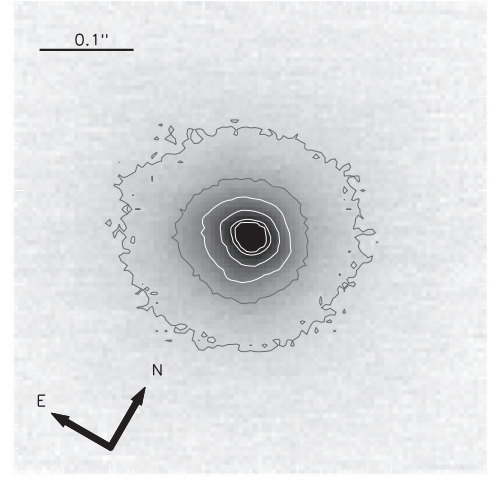

(viii) $S D S S J 1516+3053$.

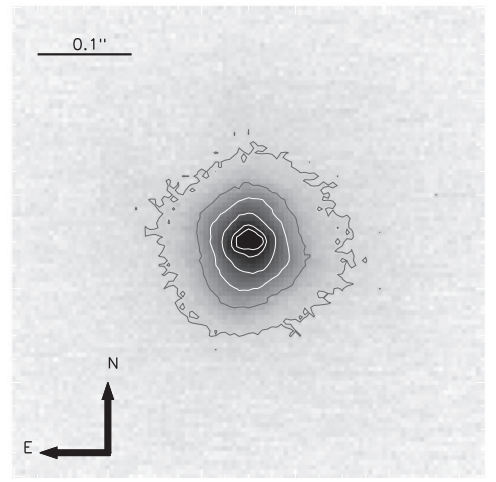

(xi) 2MASS J1711+2232.

Figure 2. (Continued.)

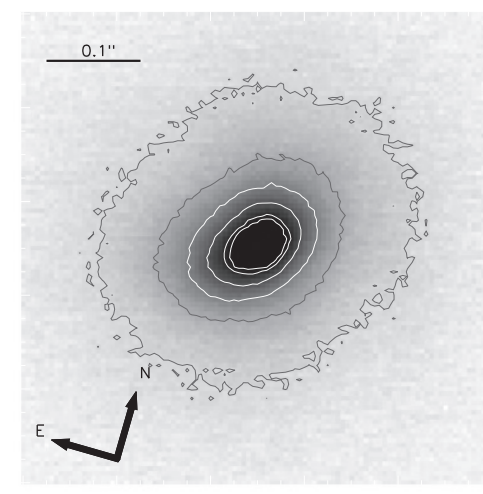

(iii) 2MASS J1209-1004.

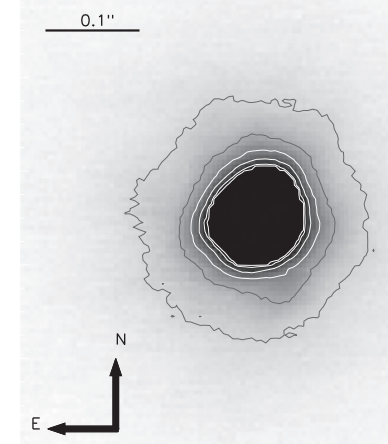

(vi) 2MASS J1428+5923.

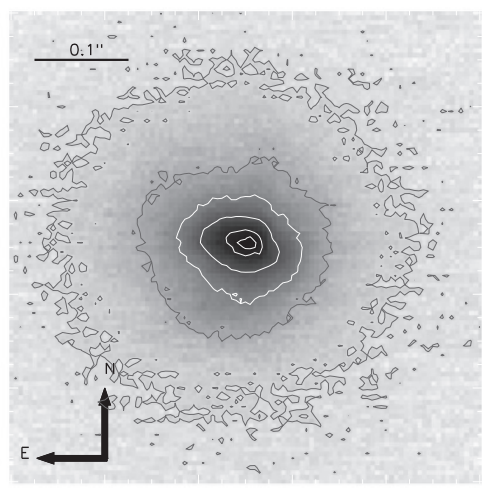

(ix) $S D S S J 1547+0336$.

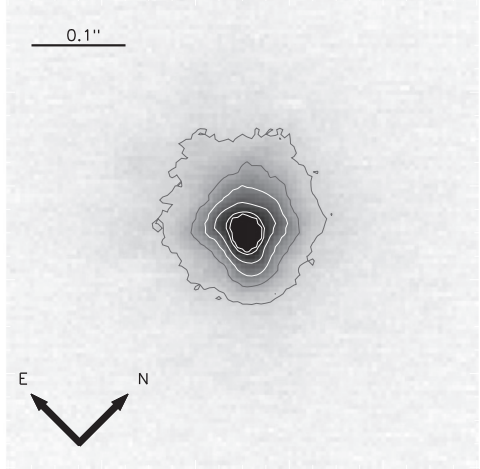

(xii) $2 M A S S J 1721+3344$. 


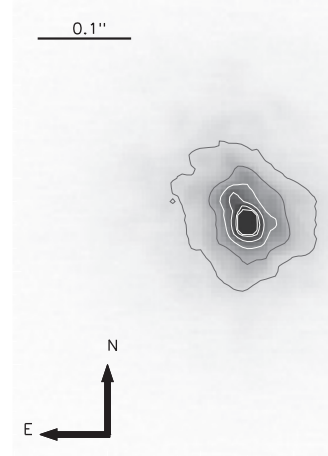

(i) $2 M A S S$ J1733-1654.

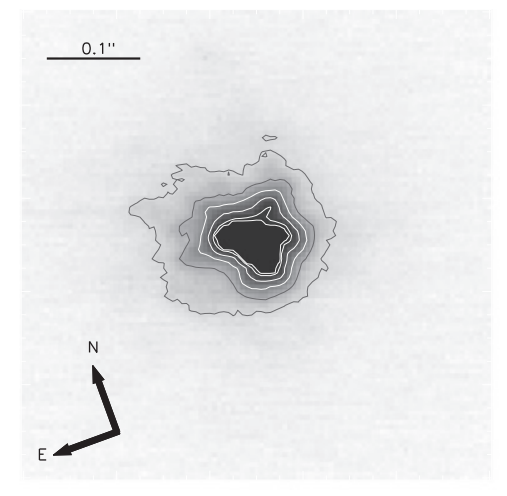

(ii) 2MASS J2002-0521.

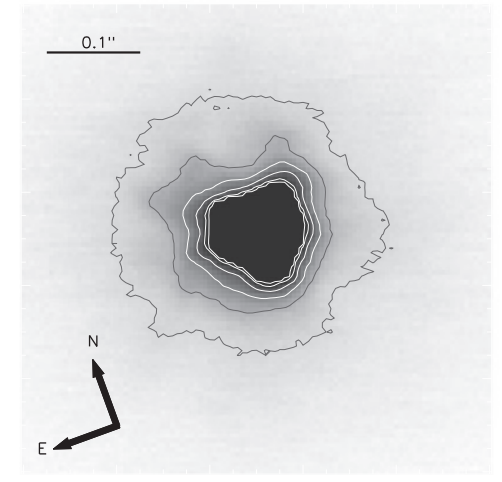

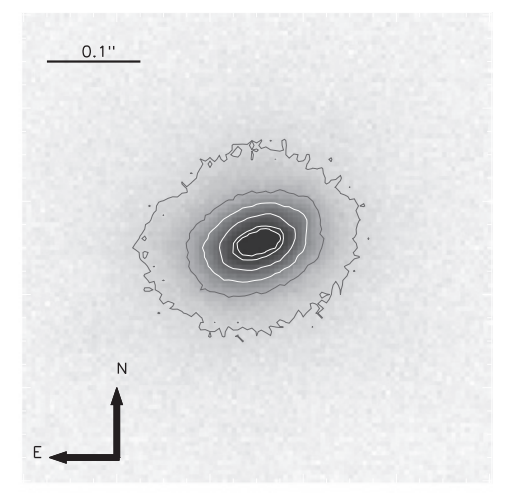

(iii) $2 M A S S$ J2126+7617.

(iv) $2 M A S S$ J2149+0603.

Figure 2. (Continued.)

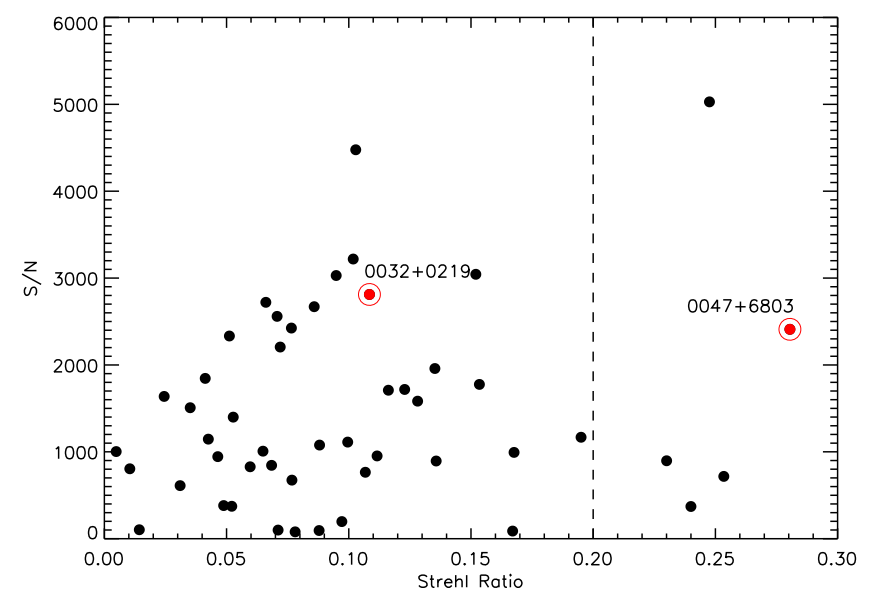

Figure 3. Strehl ratio vs. signal-to-noise ratio of all $H$ band observations (black dots). The two representative sources used in the empirical sensitivity curves are marked in red. The three groups are separated by the dashed lines. See Table 1 for the list of objects.

at an angular separation of $139 \pm 14$ mas in NIRC2 LGS-AO observations, which has been confirmed at a slightly wider offset (angular separation $197 \pm 3$ mas, projected separation $1.18 \pm 0.21 \mathrm{AU}$ ) with $\Delta H=3.85 \pm 0.11 \mathrm{mag}$ in follow-up observations (Burgasser et al. 2015b). Our analysis does not resolve the companion to limits of 500 mas and $3 \mathrm{AU}$, beyond the separation reported in that study.

\subsubsection{SDSS J080531.84+481233.0}

SDSS J0805+4812 is a blue L dwarf discovered by Hawley et al. (2002), and a spectral binary of L4.5 and T5 components (Burgasser 2007b). This source shows astrometric variability with an amplitude of 15 mas (Dupuy \& Liu 2012). Our LGSAO observations show an elongated PSF that we attribute to tip-tilt correction errors, but no resolved companion. Dupuy \& Liu (2012) estimate a semimajor axis of 40-100 mas assuming a mass ratio $q=0.55-0.88$ and from the measured parallactic distance of $22.9 \pm 0.6 \mathrm{pc}$, they infer a projected separation of 0.9-2.3 AU. Our observations do not resolve this system to limits of 164 mas and 3.8 AU, both consistent with the Dupuy \& Liu (2012) estimates.

\subsubsection{MASS J11061197+2754225}

The T2.5 2MASS J1106+2754 was first discovered by Looper et al. (2007) and later observed with NIRC2 with LGSAO in 2006 June, but was unresolved (Looper et al. 2008b). B10 identified it as a spectral binary of T0.0 \pm 0.2 and $\mathrm{T} 4.5 \pm 0.2$ components due to its $\mathrm{CH}_{4}$ absorption feature in the $H$ band, and ruled out a separation greater than $1.5 \mathrm{AU}$ based on Keck imaging. Manjavacas et al. (2013) finds that this source is $\sim 1$ mag overluminous and determined a parallactic distance of $20.6_{-1.2}^{+1.0} \mathrm{pc}$. Our LGS-AO observations were unable to resolve this source, implying upper limits of 74 mas and $1.5 \mathrm{AU}$, the same constraint as that reported by Burgasser et al. (2010). 

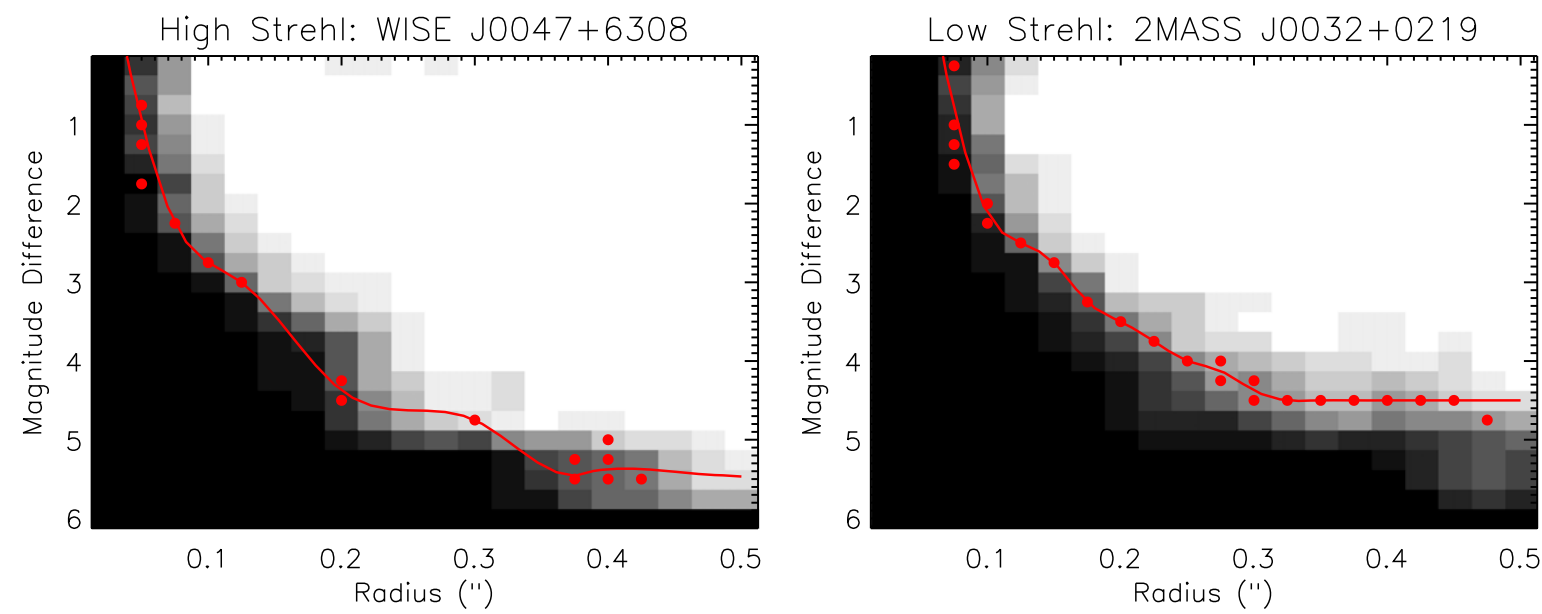

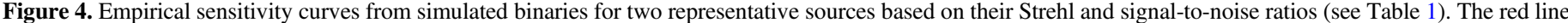
delimits the detections of secondaries (white) from non-detections (black).

\subsubsection{MASS J12095613-1004008}

2MASS J1209-1004 was first discovered by Burgasser et al. (2004) and is the T3 spectral standard (Burgasser et al. 2006a). Liu et al. (2010) resolved the system with NIRC2 and LGS-AO in the $J$ band, and estimated component types of T2.0 \pm 0.5 and $\mathrm{T} 7.5 \pm 0.5$ based on photometry. The mass ratio of this binary is estimated to be $q=0.5$, which is unusually small for brown dwarf binaries (Bouy et al. 2003; Burgasser 2007a). Liu et al. (2010) found an angular separation of $151 \pm 13$ mas at a position angle of $314^{\circ} \pm 5^{\circ}$ with a magnitude difference of $\Delta H=2.8 \pm 0.3 \mathrm{mag}$. Its parallactic distance $d=21.8 \pm 0.5 \mathrm{pc}$ (Dupuy \& Liu 2012) leads to a projected separation of $3.3 \pm 0.3 \mathrm{AU}$. This source was not resolved in our $H$ band LGS-AO image, with limits of 95 mas and 2.1 AU. In this case, our observation should have detected the companion, suggesting that orbital motion may have moved into closer projected alignment, or that the companion could be the source of the elongation of the PSF to the South East.

\subsection{Resolved Binaries}

\subsubsection{MASS J13411160-30525049}

The L3 2MASS 1341-3052 was first discovered by Reid et al. (2008) in the Two Micron All Sky Survey (2MASS; Cutri et al. 2003) and later identified as a spectral binary candidate with $\mathrm{L} 1.0 \pm 0.5$ and $\mathrm{T} 6.0 \pm 1.0$ components (BG14). Our NIRC2 observations resolve the source with an angular separation of $279 \pm 17$ mas at a position angle of $317^{\circ} .9 \pm$ 0 .6. The original template matching analysis assumed relative spectral fluxes on the Looper et al. (2008a) absolute magnitude to spectral type relation. We repeated this analysis using the relative photometry in all three $J H K_{s}$ bands to scale and select binary templates in a similar fashion as described in Burgasser et al. (2011a). Our revised template fit analysis resulted in component spectral types of L $2.5 \pm 1.0$ and T6.0 \pm 1.0 ; i.e., we infer a primary classification more consistent with the combined-light optical classification.

2MASS J1341-3052 is the only one of the three resolved systems that does not have a parallax measurement. We estimated its distance using the calculated component apparent magnitudes with the Dupuy \& Liu (2012) spectral type to absolute magnitude relation and component spectral types from template fitting. Uncertainties were propagated from spectral type, apparent magnitudes and spectral type relation, accordingly. We calculated one distance per filter and then obtained the weighted average distance from the MKO $J H K_{s}$ filters. The distances for both components $(29 \pm 3 \mathrm{pc}$ for the primary and $31 \pm 6 \mathrm{pc}$ for the secondary) were consistent across filters. The uncertainty-weighted average yields a distance estimate of 29 \pm 3 pc. From this we infer a projected separation of 8.1 $\pm 0.5 \mathrm{AU}$.

A spectral type versus absolute magnitude plot is shown in Figure 6, using the Dupuy \& Liu (2012) parallax sample of 259 objects as a reference. The primary absolute magnitude is anchored to the Dupuy \& Liu (2012) relation due to the lack of a trigonometric distance, while the secondary absolute magnitude was derived from the primary's using the measured relative magnitude. In the $J$ band, the secondary adds 0.09 mags to the primary, barely enough to make the combined absolute magnitude look like an outlier. In the $K$ band, the secondary adds 0.02 mags to the primary, so it appears as if the secondary absolute magnitude also falls on the relation and the combined magnitude is within the $+1 \sigma$ curve. This source could not have been detected as an overluminous binary candidate because of the late type of the secondary. The properties of all three resolved binary systems are summarized in Table 4.

\subsubsection{SDSS J151114.66+060742.9}

The T0 SDSS J1511+0607 was discovered by Chiu et al. (2006) in the Sloan Digital Sky Survey (SDSS; AdelmanMcCarthy et al. 2007). This source was identified as a "strong" binary candidate in B10, and found to be $1 \mathrm{mag}$ overluminous for its spectral type in $M_{\mathrm{JHK}}$ by Faherty et al. (2012). Multiband imaging with NIRC2 on 2009 August 15 resolved the system into two components separated by $108 \pm 11$ mas at a position angle of $335^{\circ} \pm 4^{\circ}$. The parallax measurement of $37 \pm 7$ mas by Faherty et al. (2012) implies a distance of $28 \pm 5 \mathrm{pc}$, which in turn corresponds to a projected separation of $2.9 \pm 0.3 \mathrm{AU}$. Including the measured relative $J H K_{s}$ magnitudes in our template fitting gives updated component spectral types of L5.0 \pm 1.0 and T5.0 \pm 0.5 .

Using the combined light magnitude, the relative magnitudes and the parallactic distance, we determined the absolute magnitudes for the components (Figure 6). While the combined 
Table 3

Projected Separation Constraints for Spectral Binaries and All Other Targets

\begin{tabular}{|c|c|c|c|c|c|c|c|c|c|c|c|}
\hline \multirow[b]{2}{*}{ Source } & \multicolumn{3}{|c|}{ Spectral Type } & \multirow[b]{2}{*}{2 MASS $\Delta J$} & \multirow[b]{2}{*}{ 2MASS $\Delta H$} & \multirow[b]{2}{*}{ 2MASS $\Delta K$} & \multirow[b]{2}{*}{ Confidence } & \multirow[b]{2}{*}{ Distance (pc) } & \multirow[b]{2}{*}{ References } & \multicolumn{2}{|c|}{ Separation } \\
\hline & Optical $^{\mathrm{a}}$ & Primary & Secondary & & & & & & & Angular (mas) & Projected (AU) \\
\hline \multicolumn{12}{|c|}{ Spectral Binaries } \\
\hline SDSS J011912.22+240331.6 & $\mathrm{T} 2.0$ & $\mathrm{~T} 0.3 \pm 0.7$ & $\mathrm{~T} 3.7 \pm 0.5$ & $-0.28 \pm 0.13$ & $0.30 \pm 0.11$ & $0.82 \pm 0.20$ & $>99 \%$ & $43 \pm 3$ & 1 & $<43$ & $<1.9$ \\
\hline SDSS J024749.90-163112.6 & $\mathrm{T} 2.0$ & $\mathrm{~L} 8.4 \pm 0.6$ & $\mathrm{~T} 5.7 \pm 0.5$ & $0.57 \pm 0.30$ & $1.62 \pm 0.33$ & $2.10 \pm 0.30$ & $100 \%$ & $40 \pm 3$ & 1 & $<96$ & $<3.8$ \\
\hline 2MASS J03440892+0111251 & L0.5 & $\mathrm{L} 0.3 \pm 0.4$ & $\mathrm{~T} 3.8 \pm 1.5$ & $2.77 \pm 0.38$ & $3.10 \pm 0.65$ & $3.50 \pm 0.70$ & $98 \%$ & $41 \pm 4$ & 1 & $<226$ & $<9.2$ \\
\hline SDSS J035104.37+481046.8 & $\mathrm{T} 1.0$ & $\mathrm{~L} 6.3 \pm 0.7$ & $\mathrm{~T} 5.4 \pm 0.9$ & $0.45 \pm 0.47$ & $1.62 \pm 0.51$ & $2.45 \pm 0.52$ & $>99 \%$ & $37 \pm 4$ & 1 & $<102$ & $<3.8$ \\
\hline 2MASS J05185995-2828372 & $\ldots$ & $\mathrm{L} 6.4 \pm 0.4$ & $\mathrm{~T} 5.6 \pm 0.5$ & $0.54 \pm 0.25$ & $1.75 \pm 0.27$ & $2.48 \pm 0.28$ & $100 \%$ & $23 \pm 1^{\mathrm{b}}$ & 4 & $<98$ & $<2.2$ \\
\hline WISE J07200320-0846513 & $\ldots$ & $\mathrm{M} 8.9 \pm 0.0$ & $\mathrm{~T} 5.2 \pm 0.7$ & $3.50 \pm 0.24$ & $4.15 \pm 0.36$ & $4.57 \pm 0.41$ & $100 \%$ & $6 \pm 1^{\mathrm{b}}$ & 2 & $<500$ & $<3.0$ \\
\hline SDSS J080531.84+481233.0 & L9.0 & $\mathrm{L} 5.3 \pm 0.1$ & $\mathrm{~T} 5.8 \pm 0.4$ & $1.92 \pm 0.17$ & $2.85 \pm 0.18$ & $3.49 \pm 0.30$ & $100 \%$ & $23 \pm 1^{\mathrm{b}}$ & 4 & $<164$ & $<3.8$ \\
\hline 2MASS J11061197+2754225 & $\mathrm{T} 2.5$ & $\mathrm{~L} 8.9 \pm 0.5$ & $\mathrm{~T} 4.2 \pm 0.4$ & $-0.24 \pm 0.14$ & $0.49 \pm 0.17$ & $1.03 \pm 0.16$ & $100 \%$ & $21 \pm 1^{\mathrm{b}}$ & 3 & $<74$ & $<1.5$ \\
\hline 2MASS J12095613-1004008 & & $\mathrm{T} 1.1 \pm 0.0$ & $\mathrm{~T} 6.0 \pm 0.4$ & $0.95 \pm 0.11$ & $1.77 \pm 0.15$ & $2.10 \pm 0.28$ & $>99 \%$ & $22 \pm 1^{\mathrm{b}}$ & 4 & $<100$ & $<2.1$ \\
\hline 2MASS J13411160-30525049 & L3.0 & $\mathrm{L} 2.3 \pm 0.6$ & $\mathrm{~T} 6.0 \pm 1.0$ & $2.68 \pm 0.08$ & $4.03 \pm 0.12$ & $4.23 \pm 0.07$ & $96 \%$ & $29 \pm 3$ & 1 & $279 \pm 17$ & $8.9 \pm 0.4$ \\
\hline SDSS J143553.25+112948.6 & $\mathrm{T} 2.0$ & $\mathrm{~L} 8.9 \pm 0.7$ & $\mathrm{~T} 5.6 \pm 0.5$ & $0.28 \pm 0.24$ & $1.38 \pm 0.28$ & $2.11 \pm 0.37$ & $>99 \%$ & $44 \pm 4$ & 1 & $<90$ & $<4.0$ \\
\hline SDSS J151114.66+060742.9 & T0.0 & $\mathrm{L} 5.2 \pm 0.9$ & $\mathrm{~T} 4.9 \pm 0.4$ & $0.25 \pm 0.13$ & $1.41 \pm 0.13$ & $2.38 \pm 0.30$ & $>99 \%$ & $28 \pm 5^{\mathrm{b}}$ & 5 & $108 \pm 11$ & $2.9 \pm 0.3$ \\
\hline SDSS J151643.01+305344.4 & T0.5 & $\mathrm{L} 7.6 \pm 0.8$ & $\mathrm{~T} 2.3 \pm 0.3$ & $-0.36 \pm 0.24$ & $0.21 \pm 0.21$ & $0.80 \pm 0.27$ & $99 \%$ & $39 \pm 4$ & 1 & $<70$ & $<2.7$ \\
\hline SDSS J154727.23+033636.3 & $\mathrm{L} 2.0$ & $\mathrm{~L} 1.8 \pm 0.2$ & $\mathrm{~T} 6.6 \pm 1.0$ & $3.32 \pm 0.53$ & $4.38 \pm 0.60$ & $4.98 \pm 0.65$ & $90 \%$ & $53 \pm 6$ & 1 & Unconstrained & Unconstrained \\
\hline 2MASS J1711457+223204 & L6.5 & $\mathrm{L} 5.5 \pm 0.5$ & $\mathrm{~T} 5.3 \pm 1.0$ & $1.21 \pm 0.41$ & $2.29 \pm 0.56$ & $3.08 \pm 0.64$ & $>99 \%$ & $30 \pm 4^{\mathrm{b}}$ & 4 & $<156$ & $<4.7$ \\
\hline 2MASS J1733423-165449 & L0.5 & $\mathrm{L} 0.1 \pm 0.2$ & $\mathrm{~T} 4.0 \pm 1.3$ & $2.76 \pm 0.30$ & $3.14 \pm 0.54$ & $3.59 \pm 0.60$ & $98 \%$ & $24 \pm 2$ & 1 & $<219$ & $<5.3$ \\
\hline SDSS J205235.31-160929.8 & $\mathrm{T} 1.0$ & $\mathrm{~L} 5.9 \pm 1.6$ & $\mathrm{~T} 2.1 \pm 0.5$ & $0.03 \pm 0.25$ & $0.44 \pm 0.30$ & $1.12 \pm 0.40$ & $>99 \%$ & $30 \pm 1^{\mathrm{b}}$ & 4 & $103 \pm 2$ & $3.2 \pm 0.5$ \\
\hline \multicolumn{12}{|c|}{ Visual Spectral Binaries } \\
\hline 2MASSI J0019457+521317 & M9.0 & $\mathrm{M} 8.5 \pm 0.2$ & $\mathrm{~T} 6.9 \pm 1.1$ & $4.82 \pm 0.54$ & $5.53 \pm 0.65$ & $5.91 \pm 0.71$ & $50 \%$ & $20 \pm 2$ & 1 & Unconstrained & Unconstrained \\
\hline 2MASS J00320509+0219017 & L1.5 & $\mathrm{L} 0.1 \pm 0.3$ & $\mathrm{~T} 6.4 \pm 1.4$ & $4.06 \pm 0.68$ & $4.87 \pm 0.83$ & $5.36 \pm 0.85$ & $49 \%$ & $33 \pm 4$ & 1 & Unconstrained & Unconstrained \\
\hline SDSS J003259.36+141036.6 & L8.0 & $\mathrm{L} 6.2 \pm 0.7$ & $\mathrm{~T} 2.4 \pm 1.9$ & $0.55 \pm 0.70$ & $1.03 \pm 0.95$ & $1.54 \pm 0.97$ & $50 \%$ & $33 \pm 6^{\mathrm{b}}$ & 4 & $<96$ & $<3.2$ \\
\hline SDSSp J023617.93+004855.0 & L6.5 & $\mathrm{L} 5.1 \pm 0.5$ & $\mathrm{~T} 1.9 \pm 1.2$ & $1.04 \pm 0.49$ & $1.33 \pm 0.59$ & $1.81 \pm 0.58$ & $7 \%$ & $39 \pm 4$ & 1 & $<95$ & $<3.7$ \\
\hline SDSS J075840.33+324723.4 & $\mathrm{T} 2.0$ & $\mathrm{~T} 2.3 \pm 0.0$ & $\mathrm{~T} 2.2 \pm 0.0$ & $-0.28 \pm 0.0$ & $-0.07 \pm 0.0$ & $0.193 \pm 0.0$ & $92 \%$ & $16 \pm 2$ & 1 & $<65$ & $<1.0$ \\
\hline SDSS J093109.56+032732.5 & L7.5 & $\mathrm{L} 7.2 \pm 0.3$ & $\mathrm{~T} 6.6 \pm 1.8$ & $2.66 \pm 0.49$ & $3.45 \pm 0.78$ & $3.87 \pm 0.91$ & $90 \%$ & $37 \pm 4$ & 1 & $<288$ & $<10.7$ \\
\hline 2MASS J09490860-1545485 & $\mathrm{T} 2.0$ & $\mathrm{~T} 1.1 \pm 0.2$ & $\mathrm{~T} 3.5 \pm 2.0$ & $0.54 \pm 1.01$ & $0.83 \pm 1.28$ & $0.83 \pm 1.26$ & $95 \%$ & $18 \pm 2^{\mathrm{b}}$ & 5 & $<101$ & $<1.8$ \\
\hline SDSS J103321.92+400549.5 & L6.0 & $\mathrm{L} 4.9 \pm 0.6$ & $\mathrm{~T} 4.4 \pm 2.2$ & $1.96 \pm 0.72$ & $2.52 \pm 1.02$ & $2.99 \pm 1.07$ & $84 \%$ & $54 \pm 6$ & 1 & $<206$ & $<11.2$ \\
\hline SDSS J112118.57+433246.5 & L7.5 & $\mathrm{L} 6.8 \pm 0.5$ & $\mathrm{~T} 5.0 \pm 1.5$ & $1.52 \pm 0.64$ & $2.21 \pm 0.80$ & $2.74 \pm 0.84$ & $95 \%$ & $52 \pm 6$ & 1 & $<164$ & $<8.4$ \\
\hline 2MASS J11582077+0435014 & sdL7 & $\mathrm{L} 6.4 \pm 0.0$ & $\mathrm{~T} 2.6 \pm 0.8$ & $1.42 \pm 0.12$ & $1.57 \pm 0.22$ & $1.98 \pm 0.26$ & $100 \%$ & $28 \pm 2$ & 1 & $<93$ & $<2.5$ \\
\hline SDSS J120602.51+281328.7 & $\mathrm{T} 3.0$ & $\mathrm{~T} 2.0 \pm 0.4$ & $\mathrm{~T} 5.0 \pm 0.8$ & $0.46 \pm 0.35$ & $1.05 \pm 0.49$ & $1.26 \pm 0.50$ & $74 \%$ & $29 \pm 3$ & 1 & $<87$ & $<2.5$ \\
\hline 2MASS J14283132+5923354 & L4.0 & $\mathrm{L} 4.4 \pm 0.7$ & $\mathrm{~T} 6.4 \pm 2.0$ & $3.30 \pm 0.72$ & $4.24 \pm 1.00$ & $4.77 \pm 1.06$ & $68 \%$ & $21 \pm 3$ & 1 & Unconstrained & Unconstrained \\
\hline 2MASS J17073334+4301304 & L0.5 & $\mathrm{M} 8.7 \pm 0.1$ & $\mathrm{~T} 6.9 \pm 0.7$ & $4.41 \pm 0.47$ & $5.28 \pm 0.51$ & $5.75 \pm 0.53$ & $60 \%$ & $35 \pm 3$ & 1 & Unconstrained & Unconstrained \\
\hline 2MASS J1721039+334415 & L3.0 & $\mathrm{L} 2.5 \pm 0.0$ & $\mathrm{~T} 3.8 \pm 2.0$ & $2.91 \pm 0.62$ & $3.10 \pm 0.99$ & $3.32 \pm 1.09$ & $99 \%$ & $19 \pm 2$ & 1 & $<270$ & $<5.1$ \\
\hline 2MASS J21265916+7617440 & T0.0 & $\mathrm{L} 8.5 \pm 1.0$ & $\mathrm{~T} 4.5 \pm 2.0$ & $0.42 \pm 0.82$ & $1.21 \pm 1.01$ & $1.75 \pm 1.03$ & $63 \%$ & $12 \pm 2$ & 1 & $<106$ & $<1.3$ \\
\hline SDSS J214956.55+060334 & M9.0 & $\mathrm{M} 8.2 \pm 0.0$ & $\mathrm{~T} 6.6 \pm 1.2$ & $4.83 \pm 0.59$ & $5.53 \pm 0.70$ & $5.91 \pm 0.74$ & $58 \%$ & $29 \pm 3$ & 1 & Unconstrained & Unconstrained \\
\hline \multicolumn{12}{|c|}{$\begin{array}{l}\text { Notes. } \\
{ }^{a} \text { Unresolved or combined optical spectral type. } \\
\text { b Parallactic distance. Otherwise, spectrophotometric distance, assuming relative magnitudes from template fitting. } \\
{ }^{c} \text { Resolved binary with measured delta magnitudes. }\end{array}$} \\
\hline
\end{tabular}




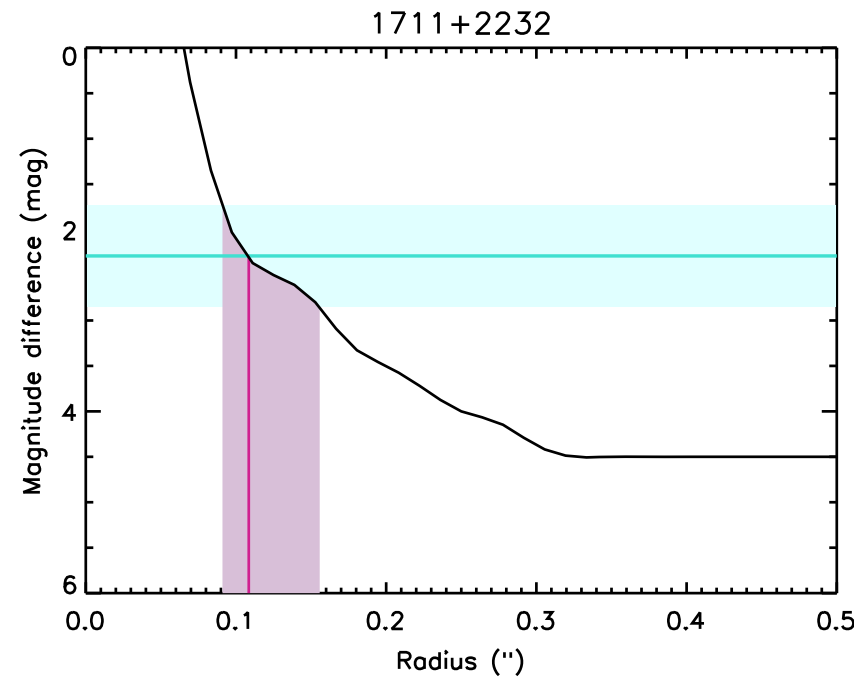

Figure 5. Example separation constraint for 2 MASS J1711+2232. Given the empirical sensitivity curve for its Strehl ratio vs. S/N group (low Strehl-low $\mathrm{S} / \mathrm{N}$, representative source: 2MASS J0032+0219) in black, and the estimated magnitude difference in cyan (uncertainties are shown as the shaded region), we can set an upper limit for an undetected secondary at the intersection (magenta line). The values for the separation constraints for all observations are reported in Table 3.

absolute magnitude of SDSS J1511+0607 clearly stands out as an outlier in spectral type to absolute magnitude plots, its components look typical. Indeed, its primary lies slightly below the Dupuy \& Liu (2012) spectral type to absolute magnitude relation.

\subsubsection{SDSS J205235.31-160929.8}

Also discovered by Chiu et al. (2006), SDSS 2052-1609 was classified as an $\mathrm{T} 1 \pm 1$ brown dwarf. B10 identified it as a spectral binary candidate with component types of L7.5 \pm 1.0 and $\mathrm{T} 2 \pm 0.5$, noting that the best fit primary was unusually blue when compared to the median $J-K_{s}$ colors of Faherty et al. (2009). Stumpf et al. (2011) was able to resolve the components with VLT/NACO, and also reported archival HST/NICMOS data which confirmed common proper motion and indicated some orbital motion. Stumpf et al. (2011) determined component spectral types by comparing the objects' $J H K_{s}$ colors to mean colors from Dwarf Archives 8 and obtained divergent results for the primary component (T0.5 \pm 0.5 and T2.5 \pm 0.5 ) as compared to those from B10. Using our NIRC2 photometry to constrain spectral template fitting, we find component spectral types of L6.0 \pm 2.0 and $\mathrm{T} 2.0 \pm 0.5$. Dupuy \& Liu (2012) find a parallactic distance of $29.5 \pm 0.7 \mathrm{pc}$. The angular separation between the components was measured to be $103 \pm 2$ mas, leading to a projected separation of $3.0 \pm 0.1 \mathrm{AU}$.

Absolute magnitudes of this source and its components were calculated from its parallactic distance and measured magnitude differences. As for the case of SDSS J1511+0607, the primary of SDSS J2052-1609 appears to be underluminous, while its secondary falls comfortably within $1 \sigma$ from the Dupuy \& Liu (2012) relation (Figure 6).

\footnotetext{
8 http://www.dwarfarchives.org
}

\section{DISCUSSION}

\subsection{A Preliminary Orbit for SDSS J2052-1609AB}

Our observations of SDSS J2052-1609AB confirm prior results by Stumpf et al. (2011) and adds to coverage of its orbital motion first detected in that study. We identified additional archival NIRC2 + LGSAO images of the system taken on 2005 October 11, 2007 April 23 (PI M. Liu) and 2010 May 1 (PI B. Biller), and analyzed these data in the same manner as described above. The resulting six epochs of relative astrometry spanning just over 4.5 years are listed in Table 5 and displayed in Figure 7. These measurements confirm the direction of motion previously identified and cover a significant fraction of the system's orbit.

To more tightly constrain the orbit of this system, we used an Markov Chain Monte Carlo (MCMC) routine with MetropolisHasting algorithm (Metropolis et al. 1953; Hastings 1970) to iteratively fit a seven-parameter orbit model to the twelve astrometric measurements (six each in relative R.A. and decl.) and parallax distance measurement $(d=29.5 \pm 0.7 \mathrm{pc}$; Dupuy $\&$ Liu 2012). The methodology used is described in detail in Burgasser et al. (2015b). The parameter vector is

$$
\boldsymbol{\theta}=\left(P, a, e, i, \omega, \Omega, M_{0}, d\right)
$$

where $P$ is the period of the orbit in years, $a$ the semimajor axis in $\mathrm{AU}, e$ the eccentricity, $i$ the inclination, $\omega$ the argument of periastron, $\Omega$ the longitude of nodes, $M_{0}$ the mean anomaly at epoch $\tau_{0}=2453654.31$ (Julian Date), and $d$ is the distance in pc. We computed an MCMC chain of $10^{7}$ parameter sets, at each step varying parameters using a normal distribution with scale factors that were allowed to vary dynamically to improve convergence. ${ }^{9}$ We applied additional constraints of 1 year $<P<100$ years and $0<e<0.6$ to eliminate improbable regions of parameter space, and constained the distance to lie within $28 \mathrm{pc}<d<31 \mathrm{pc}$; our parameter chain was largely insensitive to these limits. Convergence of the chain was monitored through autocorrelation of parameters and evolution of divergence in sequential subchains, and acceptance rates were typically $0.5 \%-1 \%$. The first $10 \%$ of the MCMC chain was removed from subsequent analysis.

Figure 7 shows the best-fit relative visual orbit compared to the measurements, which is an acceptable fit $\left(\chi^{2}=12.05\right.$ for 6 degrees of freedom). Table 6 lists the best-fit orbital parameters, as well as median values and $16 \%$ and $84 \%$ quartiles, while Figure 8 displays the distributions and correlations of $P, a, e, i$ and $M_{\mathrm{tot}}=a^{3} / P^{2}$, the total system mass in Solar units. All of the parameters are reasonably welldetermined despite the limited phase coverage, although there are strong correlations between $P, a, i$ and $e$ and a hint of a secondary solution (double-peaked distributions). From the primary solution, we estimate an orbit period of $33_{-2}^{+4}$ years and total system mass of $0.0823_{-0.0017}^{+0.0037} M_{\odot}$, which is consistent with the lower limit of $0.074 M_{\odot}$ proposed by Stumpf et al. (2011) assuming a circular orbit. Indeed, the orbit of SDSS J2052-1609AB appears to be fairly circular $\left(0.014_{-0.010}^{+0.023}\right)$ and significantly inclined to the line of sight $\left(45_{-2^{\circ}}^{\circ}\right)$. The best fit

\footnotetext{
9 We used an initial scale factor set $\boldsymbol{\beta}=$ (5 years, $0.5 \mathrm{AU}, 0.2,20^{\circ}, 20^{\circ}, 20^{\circ}$, $20^{\circ}, 0.7 \mathrm{pc}$ ), but if a parameter $\theta_{\mathrm{j}}$ did not change in 1000 iterations, $\beta_{\mathrm{j}}$ was changed to 3 times the standard deviation of the previous (up to 100) changed values.
} 

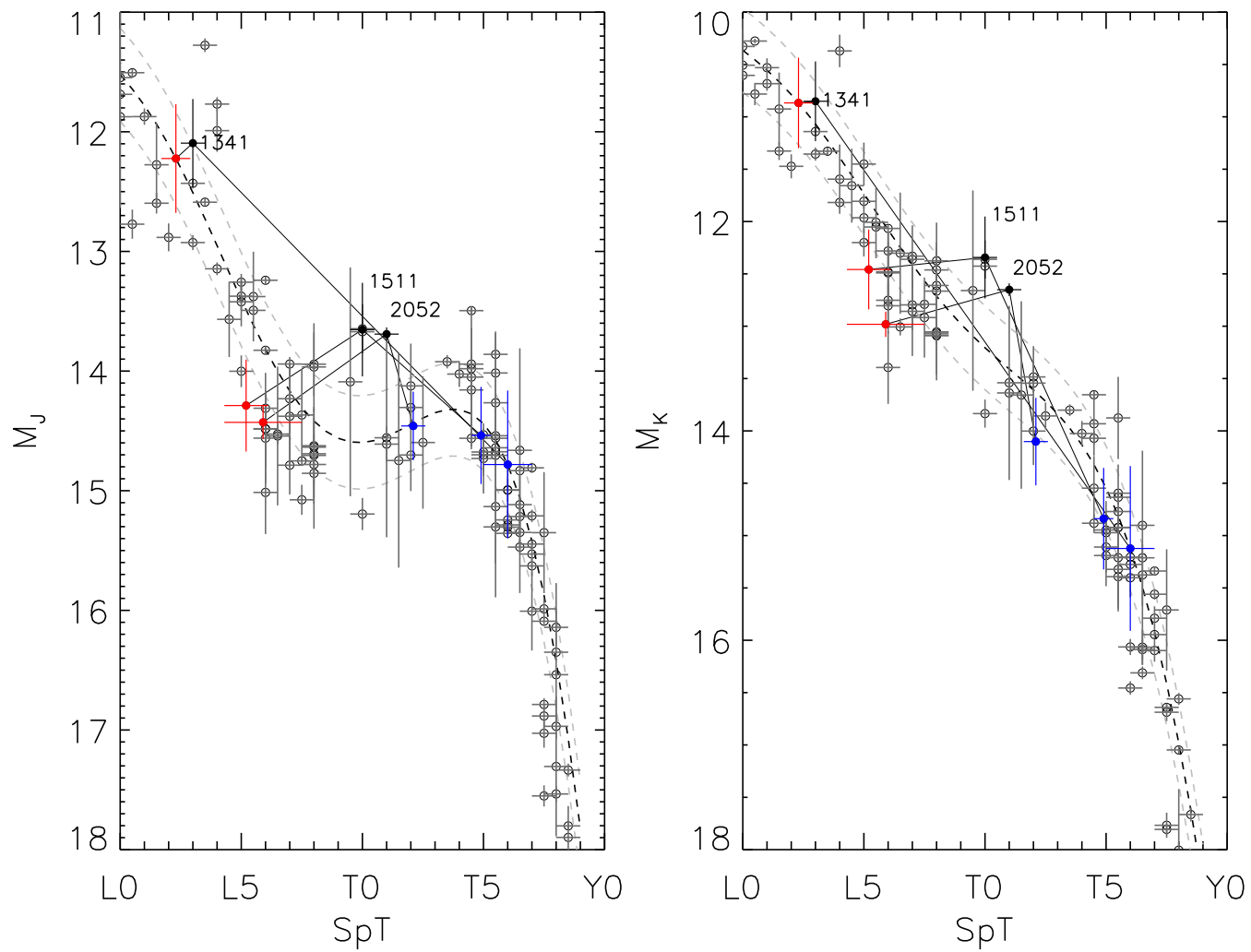

Figure 6. Absolute magnitudes in $J$ and $K_{s}$ for the three resolved binaries (black dots) and the Dupuy \& Liu (2012) parallax sample (gray circles) shown with the Dupuy \& Liu (2012) spectral type to absolute magnitude relation (black dashed line) and its $1 \sigma$ outliers (gray dashed lines). Only SDSS J1511+0607 and SDSS J2052-1609 have parallaxes reported in the literature. The three binaries are split into component spectral types, where the primaries for the two objects with parallaxes lie on the faint end of the absolute magnitude relation for their spectral type (red dots), and the secondaries (blue dots) are all within 1- $\sigma$ from the relation.

Table 4

Properties of Three Resolved Binary Systems

\begin{tabular}{lccc}
\hline \hline & 2MASS & SDSS & SDSS \\
Parameter & $\mathrm{J} 1341-3052$ & $\mathrm{~J} 1511+0607$ & $\mathrm{~J} 2052-1609$ \\
\hline Primary SpT & $\mathrm{L} 2.5 \pm 1.0$ & $\mathrm{~L} 5.5 \pm 1.0$ & $\mathrm{~L} 6.0 \pm 2.0$ \\
Secondary & $\mathrm{T} 6.0 \pm 1.0$ & $\mathrm{~T} 5.0 \pm 0.5$ & $\mathrm{~T} 2.0 \pm 0.5$ \\
$\quad$ SpT & & & \\
$\Delta J$ & $2.68 \pm 0.08$ & $0.25 \pm 0.13$ & $0.03 \pm 0.25$ \\
$\Delta H$ & $4.03 \pm 0.12$ & $1.41 \pm 0.13$ & $0.44 \pm 0.30$ \\
$\Delta K_{s}$ & $4.23 \pm 0.07$ & $2.38 \pm 0.30$ & $1.12 \pm 0.40$ \\
Distance (pc) & $29 \pm 3^{\mathrm{a}}$ & $28 \pm 5^{\mathrm{b}}$ & $30 \pm 1^{\mathrm{c}}$ \\
Ang. & $279 \pm 17$ & $108 \pm 11$ & $103 \pm 2$ \\
$\quad$ Sep. (mas) & $8.1 \pm 0.5$ & $2.9 \pm 0.3$ & $3.0 \pm 0.1$ \\
Proj. & & & $68.4 \pm 1.1$ \\
$\quad$ Sep. (AU) & $317.9 \pm 0.6$ & $335.0 \pm 4.3$ & 2455058.94 \\
PA ( ${ }^{\circ}$ ) & 2456671.15 & 2455058.77 & \\
Epoch (JD) & & & \\
\hline
\end{tabular}

Notes.

a This paper.

b Faherty et al. (2012).

c Dupuy \& Liu (2012).

template fitting results suggest component spectral types of L5.8 \pm 1.8 and T2.1 \pm 0.5 . Assuming effective temperatures corresponding to these spectral types $(1544 \pm 181 \mathrm{~K}$ for the primary and $1248 \pm 101 \mathrm{~K}$ for the secondary; Stephens et al. 2009), and using the evolutionary models of Saumon \& Marley (2008) to estimate age-dependent component masses, we estimate an age of 0.4-1.4 Gyr for this system, where the range accounts for the total mass uncertainty, effective temperature uncertainties, and cloud effects on brown dwarf evolution. Observations over the next decade should greatly improve the mass and orbit constraints on this system, and resolved spectroscopy should make it possible to critically test evolutionary models (e.g., Konopacky et al. 2010; Dupuy et al. 2014).

\subsection{Estimated Orbital Parameters for 2MASS J1341-3052 and SDSS $1511+0607$}

For 2MASS J1341-3052 and SDSS J1511+0607 only a single epoch of astrometry is available. Hence, we performed a simple Monte Carlo simulation to find the distributions of likely semimajor axes and periods for these systems. Following the procedure described in Burgasser et al. (2015a), we created random uniformly distributed vectors for eccentricity $0<\epsilon<0.6$ (Dupuy \& Liu 2011), inclination $0<\sin i<1$, longitude of ascending node $0<\Omega<2 \pi$, argument of periapse $0<\omega<2 \pi$, and mean anomaly angle $0<M<2 \pi$ for $10^{5}$ hypothetical orbits with a fixed semimajor axis of $a=1 \mathrm{AU}$. We numerically solved the Kepler equation to find the eccentric anomaly, calculated the Thiele-Innes constants (Innes 1907; van den Bos 1927), and found the $x$ and $y$ projected positions on the sky leading to the total projected separation, $r_{\text {tot }}$. The distributions of semimajor axes for the resolved systems were inferred by transforming variables:

$$
a=(1 \mathrm{AU}) \times \frac{\rho d}{r_{\mathrm{tot}}}
$$


Table 5

Resolved Separation Measurements for SDSS J2052-1609AB

\begin{tabular}{|c|c|c|c|c|c|}
\hline UT Date & JD & Instrument & $\begin{array}{c}\Delta \alpha \\
(\mathrm{mas})\end{array}$ & $\begin{array}{c}\Delta \delta \\
(\mathrm{mas})\end{array}$ & References \\
\hline 2005 Oct 11 & 2453654 & Keck/NIRC2 & $25.4 \pm 1.2$ & $114.3 \pm 1.4$ & 1,2 \\
\hline 2007 Apr 23 & 2454213 & Keck/NIRC2 & $54.6 \pm 2.1$ & $88.8 \pm 1.5$ & 1,2 \\
\hline 2009 Jun 19 & 2455002 & VLT/NACO & $93.1 \pm 1.0$ & $38.9 \pm 0.9$ & 3 \\
\hline 2009 Aug 15 & 2455058 & Keck/NIRC2 & $95.7 \pm 0.7$ & $38.1 \pm 0.7$ & 1 \\
\hline 2010 May 01 & 2455317 & Keck/NIRC2 & $103.4 \pm 1.7$ & $20.6 \pm 1.5$ & 1,4 \\
\hline
\end{tabular}

References. (1) This paper, (2) NIRC2 PI M. Liu, (3) Stumpf et al. (2011), (4) NIRC2 PI B. Biller.

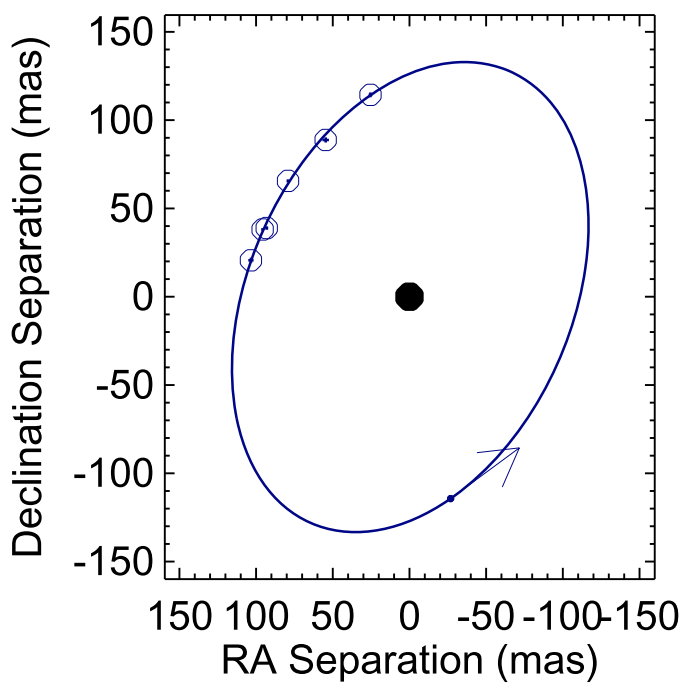

Figure 7. Visual orbit of SDSS J2052-1609AB based on MCMC analysis of separation measurements reported here and in Stumpf et al. (2011). The orbital motion of the secondary (blue line) relative to the primary (black dot at the origin) is shown projected on the sky, with the arrow indicating the direction of orbital motion at periaps $\left(M=0^{\circ}\right)$. Error bars are plotted but indiscernible on this scale.

Table 6

Orbital Analysis of SDSS J2052-1609AB Relative Astrometry

\begin{tabular}{lcc}
\hline \hline Parameter & Best & Median \\
\hline Best $\chi^{2}($ dof $)$ & $12.05(6)$ & $\ldots$ \\
$P^{\mathrm{a}}($ year $)$ & 32 & $33_{-2}^{+4}$ \\
$a(\mathrm{AU})$ & 4.4 & $4.5_{-0.2}^{+0.5}$ \\
$e^{\mathrm{a}}$ & 0.005 & $0.014_{-0.010}^{+0.023}$ \\
$i\left(^{\circ}\right)$ & 45 & $45_{-3}^{+5}$ \\
$\omega\left(^{\circ}\right)$ & 98 & $100_{-13}^{+15}$ \\
$\Omega\left(^{\circ}\right)$ & 327 & $327_{-4}^{+3}$ \\
$M_{0}\left({ }^{\circ}\right)$ & 318 & $313_{-8}^{+15}$ \\
$d^{\mathrm{a}}(\mathrm{pc})$ & 30.5 & $30.7_{-0.4}^{+0.2}$ \\
$M_{\text {tot }}\left(M_{\odot}\right)$ & 0.081 & $0.0823_{-0.0017}^{+0.0037}$ \\
\hline
\end{tabular}

Note.

${ }^{\text {a }}$ Parameter was constrained to a limited value range in MCMC analysis.

where $a$ is the semimajor axis in $\mathrm{AU}, \rho$ is the angular separation in arc seconds, $d$ is the distance to the system in parsecs and $r_{\text {tot }}$ is in AU. The observed projected separation, $r_{\mathrm{obs}}=\rho d$, constrains the array of allowed orbits, $r_{\text {tot }}$, and as a result we arrive at a distribution of probable semimajor axes, $a$.
The cumulative probability distributions for the semimajor axes of 2MASS J1341-3052 and SDSS J1511+0607 are shown in Figure 9. The most likely semimajor axes are demarcated by a dashed red line and the central 68\% ( $\pm 1 \sigma$ equivalent) of the data are shaded in lavender. We estimated the periods for these orbits in years assuming $P^{2}=a^{3} / M_{\mathrm{tot}}$, with $M_{\mathrm{tot}}=M_{1}+M_{2}$ in Solar masses estimated from the models of Baraffe et al. (2003) for ages of 0.5, 1, 5 and $10 \mathrm{Gyr}$ (Table 7). For 2MASS J1341-3052, we obtain most likely semimajor axis and period of $8.6_{-1.8}^{+5.2} \mathrm{AU}$ and $63-85$ years for decreasing ages, while for SDSS J1511+0607 the most likely semimajor axis and periods are $3.4_{-0.8}^{+1.8} \mathrm{AU}$ and $15-21$ years.

\subsection{On the Frequency of Short Period VLM Binaries}

Starting from a sample of LGS-AO observations of 43 brown dwarfs, we resolved 3 of 17 spectral binary candidates and none of the other targets. 2MASS J1733-1654 has a particularly asymmetrical PSF shape which could indicate the presence of a marginally resolved companion NE of the primary, but this is inconclusive from our images. The fraction of resolved systems from the spectral binary sample is $3 / 17=18_{-6}^{+13} \%$ (binomial uncertainties), which is consistent with the observed binary fractions reported in the literature from imaging programs (10\%-20\%; Bouy et al. 2003; Close et al. 2003; Basri \& Reiners 2006; Allen 2007; Burgasser 2007a; Kraus \& Hillenbrand 2012). Among the spectral binaries, 2MASS J0518-2828, WISEP J0720-0846, SDSS J0805+4812, 2MASS J1106+2754 and 2MASS J1209-1004 are known binaries unresolved in our images. This indicates a minimum binary fraction for spectral binaries in this sample of $(3+5) / 17=47_{-11}^{+12} \%$. This is considerably higher than typical VLM binary search samples and indicates that the spectral binary sample is positively biased toward binaries. The fact that over half of the known binaries in this sample are not resolved suggests that imaging programs are similarly missing binaries, and that the true binary fraction may be significantly higher than what is currently reported. Note that the fraction reported here remains a lower limit; an unknown number of the 9 unresolved and unconfirmed spectral binaries may be true binaries with separations $\lesssim 1.5-9.2$ AU.

Of the 12 confirmed spectral binaries to date (Table 8), about half have been unresolved in reported LGS-AO imaging, which is roughly consistent with the 3 resolved and 5 unresolved binaries in our sample. While follow-up of unresolved systems is more time-consuming and resource-intensive (RV and astrometric monitoring), we speculate that the number of unresolved but confirmed spectral binaries will increase as 


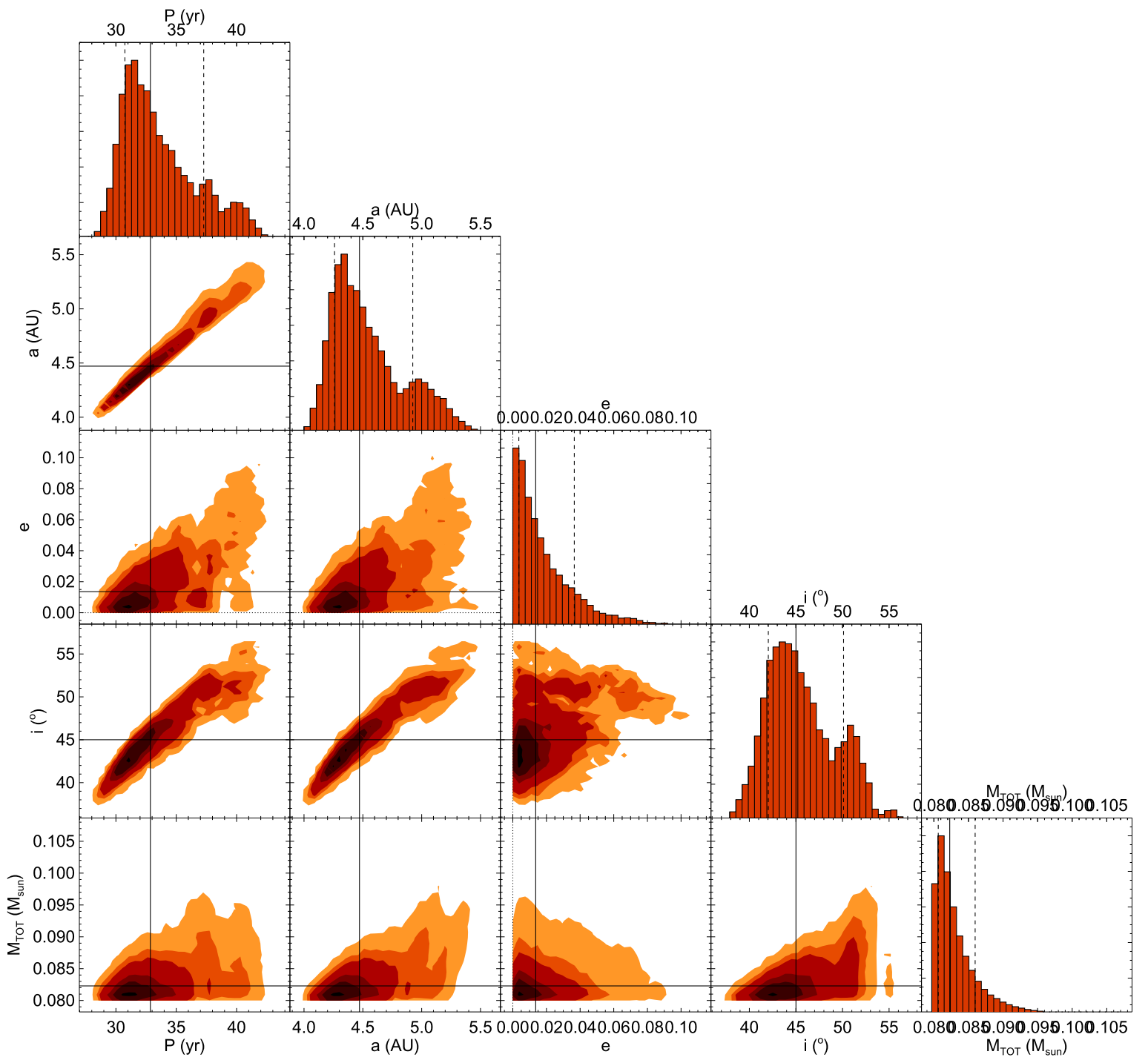

Figure 8. Parameter distributions and correlations (triangle plot) for period $(P)$, semimajor axis $(a)$, eccentricity $(e)$, inclination $(i)$, and total system mass $\left(M_{\text {tot }}\right)$ based on our MCMC orbital analysis of SDSS J2052-1609AB. Contour plots show $\chi^{2}$ distributions as a function of parameter pairs, highlighting correlations. Normalized histograms at the ends of rows are marginalized over all other parameters. Median values are indicated by solid lines in all panels, and $16 \%$ and $84 \%$ quantiles are indicated by dashed lines in the histograms.

follow-up is completed. A high incidence of unresolved but confirmed spectral binaries implies a high incidence of currently unresolved binaries in general. For example, if all of the unresolved spectral binaries in our sample actually are binaries, this would indicate a ratio of unresolved-to-resolved systems of $4.7: 1$. Given that the resolved binary fraction is roughly $15 \%$, this rate of unresolved pairs would imply an overall binary fraction of over $60 \%$. It is more likely that the current pool of spectral binary candidates contains some number of contaminants, such as blue L dwarfs (BG14) and variable brown dwarfs (Radigan et al. 2012; Khandrika et al. 2013) which will need to be identified through more detailed spectral and photometric variability analysis. Nevertheless, it is important to note that all 17 spectral binary systems studied here have resolved or upper limit separations near or below the peak of the resolved binary separation of VLM dwarfs (Allen 2007). This is strong evidence that a large number of VLM binaries are being missed in current imaging surveys.

\section{SUMMARY}

We have observed 43 late-M, L and T dwarfs with Keck/ NIRC2 LGS-AO, including 17 spectral binary candidates with high resolution Keck/NIRC2 LGS-AO imaging. Three sources were resolved: 2MASS J1341-3052, SDSS J1511+0607 and SDSS J2052-1609, while five other confirmed binaries were unresolved. Only one of our spectral binary candidates, 2MASS J1733-1654, has a candidate faint companion on the images, requiring confirmation. We used relative photometry to update the estimated component spectral types of our resolved systems. For SDSS J2052-1609, we combined our observations with those of Stumpf et al. (2011) and archival data to make a preliminary determination of orbital parameters, finding a period of $33_{-2}^{+4}$ years and system mass of $0.0823_{-0.0017}^{+0.0037} M_{\odot}$ consistent with a relatively young system $(0.4-1.4 \mathrm{Gyr})$. For 2MASS J1341-3052 and SDSS J1511+0607, we estimated their most likely semimajor axes and periods based on their observed angular separations, distances and estimated masses. 2MASS J1341-3052 has a projected separation of 

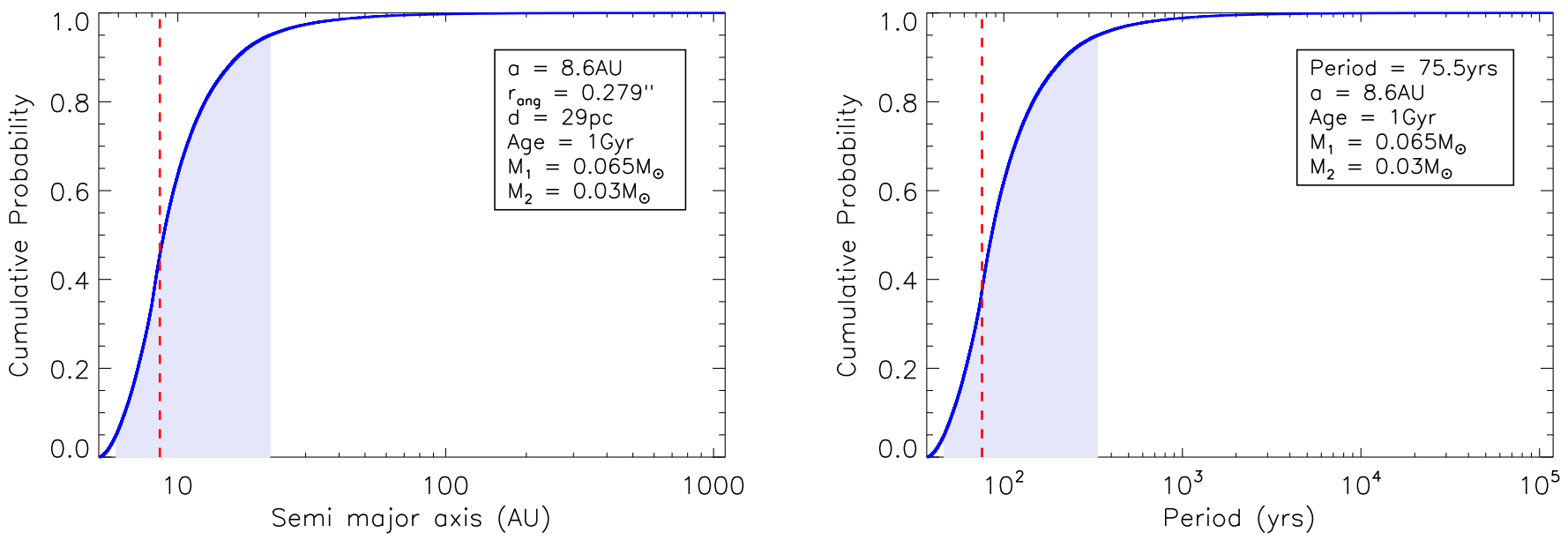

(a) 2MASS J1341-3052.
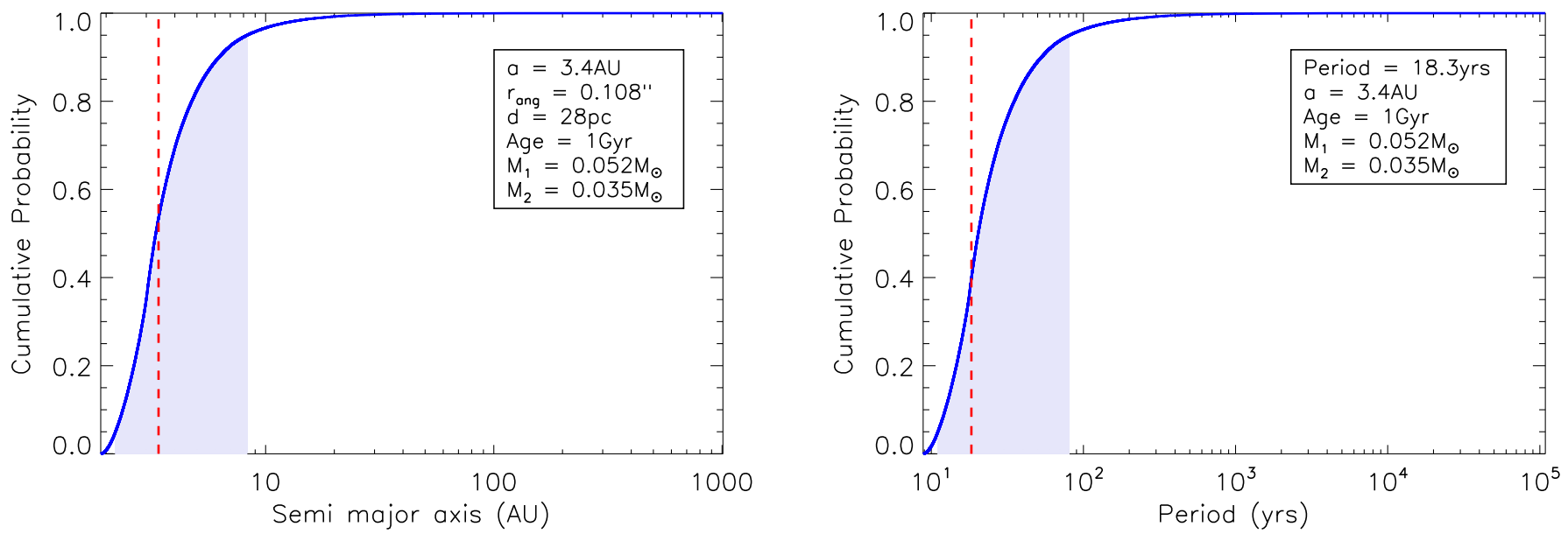

(b) SDSS J1511+0607.

Figure 9. Cumulative probability distribution of possible semimajor axes and periods for two of the resolved sources from a Monte Carlo simulation for an age of 1 Gyr, using one single observation for projected separation from the LGS-AO images in each case. The simulation parameters are shown on the box in the upper right corner. The most likely semimajor axis and period is represented by the dotted red line. The shaded regions indicate the central $68 \%$ ( $\pm 1 \sigma$ equivalent) of data points.

Table 7

Estimated Masses and Orbit Results from Monte Carlo Simulation

\begin{tabular}{|c|c|c|c|c|c|}
\hline System & Age (Gyr) & Primary Mass $\left(M_{\odot}\right)$ & Secondary Mass $\left(M_{\odot}\right)$ & Semimajor Axis (AU) & Period (years) \\
\hline 2MASS J1341-3052AB & 0.5 & $0.052 \pm 0.005$ & $0.022 \pm 0.005$ & $8.6_{-1.8}^{+5.2}$ & $85_{-20}^{+104}$ \\
\hline $\mathrm{SpT}=\mathrm{L} 2.5 \pm 1.0$ and $\mathrm{T} 6.0 \pm 1.0$ & 1.0 & $0.065 \pm 0.004$ & $0.030 \pm 0.007$ & $8.6_{-1.8}^{+5.3}$ & $71_{-18}^{+91}$ \\
\hline Primary $T_{\text {eff }}=1904 \pm 165 \mathrm{~K}$ & 5.0 & $0.075 \pm 0.002$ & $0.054 \pm 0.007$ & $8.6_{-1.8}^{+5.3}$ & $64_{-15}^{+79}$ \\
\hline Secondary $T_{\text {eff }}=1027 \pm 144 \mathrm{~K}$ & 10 & $0.075 \pm 0.001$ & $0.061 \pm 0.006$ & $8.6_{-1.8}^{+5.2}$ & $63_{-14}^{+76}$ \\
\hline SDSS J1511+0607AB & 0.5 & $0.041 \pm 0.004$ & $0.026 \pm 0.003$ & $3.4_{-0.8}^{+1.8}$ & $21_{-5}^{+25}$ \\
\hline $\mathrm{SpT}=\mathrm{L} 5.0 \pm 1.0$ and $\mathrm{T} 5.0 \pm 0.5$ & 1.0 & $0.052 \pm 0.005$ & $0.035 \pm 0.004$ & $3.4_{-0.9}^{+1.8}$ & $18_{-5}^{+22}$ \\
\hline Primary $T_{\text {eff }}=1617 \pm 139 \mathrm{~K}$ & 5.0 & $0.070 \pm 0.002$ & $0.059 \pm 0.004$ & $3.4_{-0.9}^{+1.7}$ & $15_{-3}^{+18}$ \\
\hline Secondary $T_{\text {eff }}=1115 \pm 107 \mathrm{~K}$ & 10 & $0.072 \pm 0.001$ & $0.065 \pm 0.003$ & $3.4_{-0.9}^{+1.8}$ & $15_{-4}^{+17}$ \\
\hline
\end{tabular}

$8.1 \pm 0.5 \mathrm{AU}$ and a period in the range of 63-85 years depending on the ages. For SDSS J1511+0607, we estimate a projected separation of $2.9 \pm 0.3 \mathrm{AU}$ and a period in the 17-25 years range.

For the remaining 14 unresolved spectral binaries we calculated separation limits based on their estimated component magnitude differences from template fitting and empirical sensitivity curves. Five of these unresolved systems are confirmed binaries with measured angular separations smaller than our upper limits, and therefore consistent. The other 9 unresolved systems have upper limits in angular separation of 0. " $04-0$ ". 28 , corresponding to projected separations limits of 1.5-9.2 AU. 
Table 8

Confirmed Spectral Binaries

\begin{tabular}{|c|c|c|c|c|c|c|c|c|c|c|}
\hline \multirow[b]{2}{*}{ Name } & \multicolumn{4}{|c|}{ Spectral Type } & \multirow[b]{2}{*}{ 2MASS- $J$} & \multirow[b]{2}{*}{$J-\mathrm{K}_{s}$} & \multirow[b]{2}{*}{$\Delta J$} & \multirow[b]{2}{*}{$\begin{array}{l}\text { Separations } \\
\text { (AU) }\end{array}$} & \multirow[b]{2}{*}{$\begin{array}{l}\text { Confirmation } \\
\text { method }^{\mathrm{a}}\end{array}$} & \multirow[b]{2}{*}{$\begin{array}{l}\text { Reference } \\
\text { SB; Conf. }\end{array}$} \\
\hline & $\begin{array}{c}\text { Combined } \\
\text { Optical }\end{array}$ & $\begin{array}{l}\text { Combined } \\
\text { NIR }\end{array}$ & Primary & Secondary & & & & & & \\
\hline SDSS J000649.16-085246.3 & M9 & $\ldots$ & $\mathrm{M} 8.5 \pm 0.5$ & $\mathrm{~T} 5 \pm 1$ & $14.14 \pm 0.04$ & $1.01 \pm 0.05$ & $3.15 \pm 0.31$ & $0.29 \pm 0.01$ & $\mathrm{RV}$ & $5 ; 5$ \\
\hline 2MASS J03202839-0446358 & M8: & L1 & $\mathrm{M} 8.5 \pm 0.3$ & $\mathrm{~T} 5 \pm 0.9$ & $12.13 \pm 0.03$ & $1.13 \pm 0.04$ & $3.50 \pm 0.20$ & $0.404 \pm 0.042$ & RV & $8 ; 3$ \\
\hline 2MASS J05185995-2828372 & $\ldots$ & $\ldots$ & $\mathrm{L} 8.6 \pm 0.3$ & $\mathrm{~T} 6.4 \pm 1.0$ & $15.98 \pm 0.10$ & $1.82 \pm 0.12$ & $0.13 \pm 0.19$ & $1.8 \pm 0.5$ & DI & $12 ; 10$ \\
\hline WISEP J072003.20-084651.2 & M9.5 & $\ldots$ & M8.9 \pm 0.0 & $\mathrm{~T} 5.2 \pm 0.7$ & $10.63 \pm 0.02$ & $1.16 \pm 0.03$ & $3.30 \pm 0.20$ & $0.84 \pm 0.17$ & DI & $4 ; 4$ \\
\hline SDSS J080531.84+481233.0 & L4 & L9.5 & $\mathrm{L} 5.1 \pm 0.4$ & $\mathrm{~T} 5.7 \pm 0.5$ & $14.73 \pm 0.04$ & $1.46 \pm 0.05$ & $1.50 \pm 0.09$ & $0.9-2.3$ & AV & $9 ; 13$ \\
\hline SDSS J092615.38+584720.9 & $\mathrm{T} 4.5$ & & $\mathrm{~T} 4.0 \pm 0.1$ & $\mathrm{~T} 5.3 \pm 0.7$ & $16.77 \pm 0.14$ & $<1.57$ & $0.40 \pm 0.20$ & $2.6 \pm 0.5$ & DI & $16 ; 10,11$ \\
\hline 2MASS J11061197+2754225 & $\ldots$ & $\mathrm{T} 2.5$ & $\mathrm{~T} 0.4 \pm 0.3$ & $\mathrm{~T} 4.0 \pm 0.8$ & $14.82 \pm 0.04$ & $1.02 \pm 0.07$ & $-0.37 \pm 0.06$ & $<2.67$ & OL & 7,$14 ; 15$ \\
\hline 2MASS J12095613-1004008 & $\ldots$ & $\mathrm{T} 3$ & $\mathrm{~T} 1.2 \pm 0.3$ & $\mathrm{~T} 5.9 \pm 0.6$ & $15.91 \pm 0.08$ & $0.85 \pm 0.16$ & $1.50 \pm 0.20$ & $4.8 \pm 0.2$ & DI & $1 ; 18$ \\
\hline 2MASS J13153094-2649513 & L5 & $\ldots$ & $\mathrm{L} 4.9 \pm 0.5$ & $\mathrm{~T} 6.1 \pm 2.1$ & $15.07 \pm 0.05$ & $1.63 \pm 0.07$ & $3.03 \pm 0.03$ & $6.6 \pm 0.9$ & DI & $6 ; 6$ \\
\hline 2MASS J13411160-30525049 & L3 & $\ldots$ & $\mathrm{L} 2.3 \pm 0.6$ & $\mathrm{~T} 6.0 \pm 1.0$ & $14.61 \pm 0.03$ & $1.53 \pm 0.04$ & $3.28 \pm 0.53$ & $7.8 \pm 0.5$ & DI & $2 ; 1$ \\
\hline SDSS J151114.66+060742.9 & $\ldots$ & $\mathrm{T} 0 \pm 2$ & $\mathrm{~L} 5.2 \pm 0.9$ & $\mathrm{~T} 4.9 \pm 0.5$ & $16.02 \pm 0.08$ & $1.47 \pm 0.13$ & $0.54 \pm 0.32$ & $2.9 \pm 0.3$ & DI & $7 ; 1$ \\
\hline SDSS J205235.31-160929.8 & $\ldots$ & $\mathrm{T} 1 \pm 1$ & $\mathrm{~L} 5.8 \pm 1.8$ & $\mathrm{~T} 2.1 \pm 0.5$ & $16.33 \pm 0.12$ & $1.21 \pm 0.19$ & $0.04 \pm 0.18$ & $3.0 \pm 0.1$ & DI & $7 ; 17$ \\
\hline
\end{tabular}

Note.

$\mathrm{RV}=$ Radial velocity variability, $\mathrm{DI}=$ Direct imaging, $\mathrm{AV}=$ Astrometric variability, $\mathrm{OL}=$ Overluminosity

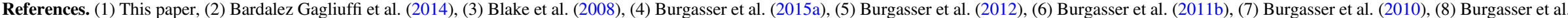

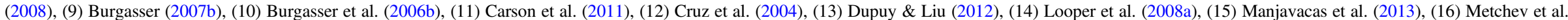
(2008), (17) Stumpf et al. (2011), (18) Liu et al. (2010). 
The binary fraction of the spectral binary candidates in this sample is $47_{-11}^{+12} \%$, significantly higher than those from prior imaging surveys (10\%-20\%; Bouy et al. 2003; Burgasser et al. 2003; Cruz et al. 2003) and the overall binary fraction (20\%25\%; Basri \& Reiners 2006; Burgasser 2007a; Joergens 2008). While this sample is clearly biased toward binary systems, the high percentage of unresolved systems suggests that there may exist a large population of very tight brown dwarf binaries that cannot be confirmed with high resolution imaging. Confirmation of the 9 remaining unresolved spectral binaries depends on high resolution RV or astrometric variability measurements that are currently ongoing. If these unresolved sources turn out to be binaries, a great advantage of their short projected separation is the high likelihood for full orbit and dynamical mass determinations. In any case, an unbiased, volume-limited sample of spectral binaries with complete follow-up is required in order to find the true underlying binary fraction.

We would like to thank our referee, Sandy Leggett, for her helpful comments. The authors thank observing assistants Jason Mcllroy, Heather Hershley, Terry Stickel, and Gary Puniwai and support astronomers Hien Tran, Luca Rizzi, Marc Kassis, and Al Conrad for their assistance during our observations. D. B. G. acknowledges funding support from the IPAC graduate fellowship. D. B. G. and A. J. B. acknowledge support from the National Aeronautics and Space Administration under Grant No. NNX15AI75G. C. R. G. was partially supported by a NASA Keck PI Data Award, administered by the NASA Exoplanet Science Institute. This research has made use of the Keck Observatory Archive (KOA), which is operated by the W. M. Keck Observatory and the NASA Exoplanet Science Institute (NExScI), under contract with the National Aeronautics and Space Administration. This publication makes use of data from the SpeX Prism Spectral Libraries, maintained by Adam Burgasser at http:// www.browndwarfs.org/spexprism; the Dwarf Archives Compendium, maintained by Chris Gelino, Davy Kirkpatrick, Mike Cushing, David Kinder and Adam Burgasser at http://Dwarf Archives.org; and the VLM Binaries Archive maintained by Nick Siegler athttp://vlmbinaries.org. The authors wish to recognize and acknowledge the very significant cultural role and reverence that the summit of Mauna Kea has always had within the indigenous Hawaiian community. We are most fortunate to have the opportunity to conduct observations from this mountain.

Facility: Keck NIRC2.

\section{REFERENCES}

Adelman-McCarthy, J. K., Agüeros, M. A., Allam, S. S., et al. 2007, ApJS, 172,634

Allen, P. R. 2007, ApJ, 668, 492

Baraffe, I., Chabrier, G., Barman, T. S., Allard, F., \& Hauschildt, P. H. 2003, A\&A, 402, 701

Bardalez Gagliuffi, D. C., Burgasser, A. J., Gelino, C. R., et al. 2014, ApJ, 794, 143

Basri, G., \& Reiners, A. 2006, AJ, 132, 663

Blake, C. H., Charbonneau, D., White, R. J., et al. 2008, ApJL, 678, L125

Bouy, H., Brandner, W., Martín, E. L., et al. 2003, AJ, 126, 1526

Burgasser, A. J. 2007a, ApJ, 659, 655

Burgasser, A. J. 2007b, AJ, 134, 1330

Burgasser, A. J. 2014, Astronomical Society of India Conf. Ser. 11, International Workshop on Stellar Spectral Libraries, 7
Burgasser, A. J., Bardalez-Gagliuffi, D. C., \& Gizis, J. E. 2011a, AJ, 141, 70 Burgasser, A. J., Burrows, A., \& Kirkpatrick, J. D. 2006a, ApJ, 639, 1095 Burgasser, A. J., Cruz, K. L., Cushing, M., et al. 2010, ApJ, 710, 1142 Burgasser, A. J., Gillon, M., Melis, C., et al. 2015a, AJ, 149, 104

Burgasser, A. J., Kirkpatrick, J. D., Cruz, K. L., et al. 2006b, ApJS, 166, 585 Burgasser, A. J., Kirkpatrick, J. D., Reid, I. N., et al. 2003, ApJ, 586, 512

Burgasser, A. J., Liu, M. C., Ireland, M. J., Cruz, K. L., \& Dupuy, T. J. 2008, ApJ, 681, 579

Burgasser, A. J., Luk, C., Dhital, S., et al. 2012, ApJ, 757, 110

Burgasser, A. J., McElwain, M. W., Kirkpatrick, J. D., et al. 2004, AJ, 127, 2856

Burgasser, A. J., Melis, C., Todd, J., et al. 2015b, arXiv:1508.06332

Burgasser, A. J., Reid, I. N., Siegler, N., et al. 2007, in Protostars and Planets V, ed. B. Reipurth, D. Jewitt \& K. Keil (Tucson, AZ: Univ. Arizona Press), 427

Burgasser, A. J., Sitarski, B. N., Gelino, C. R., Logsdon, S. E., \& Perrin, M. D. 2011b, ApJ, 739, 49

Carson, J. C., Marengo, M., Patten, B. M., et al. 2011, ApJ, 743, 141

Chiu, K., Fan, X., Leggett, S. K., et al. 2006, AJ, 131, 2722

Close, L. M., Siegler, N., Freed, M., \& Biller, B. 2003, ApJ, 587, 407

Cruz, K. L., Burgasser, A. J., Reid, I. N., \& Liebert, J. 2004, ApJL, 604, L61

Cruz, K. L., Reid, I. N., Liebert, J., Kirkpatrick, J. D., \& Lowrance, P. J. 2003, AJ, 126, 2421

Cutri, R. M., Skrutskie, M. F., van Dyk, S., et al. 2003, yCat, 2246, 0

Day-Jones, A. C., Marocco, F., Pinfield, D. J., et al. 2013, MNRAS, 430, 1171

Dupuy, T. J., \& Liu, M. C. 2011, ApJ, 733, 122

Dupuy, T. J., \& Liu, M. C. 2012, ApJS, 201, 19

Dupuy, T. J., Liu, M. C., \& Ireland, M. J. 2014, ApJ, 790, 133

Duquennoy, A., \& Mayor, M. 1991, A\&A, 248, 485

Faherty, J. K., Burgasser, A. J., Cruz, K. L., et al. 2009, AJ, 137, 1

Faherty, J. K., Burgasser, A. J., Walter, F. M., et al. 2012, ApJ, 752, 56

Fischer, D. A., \& Marcy, G. W. 1992, ApJ, 396, 178

Geißler, K., Metchev, S., Kirkpatrick, J. D., Berriman, G. B., \& Looper, D. 2011, ApJ, 732, 56

Gelino, C. R., \& Burgasser, A. J. 2010, AJ, 140, 110

Hastings, W. K. 1970, Biometrika, 57, 97

Hawley, S. L., Covey, K. R., Knapp, G. R., et al. 2002, AJ, 123, 3409

Innes, R. T. A. 1907 , Obs, 30, 310

Joergens, V. 2008, A\&A, 492, 545

Khandrika, H., Burgasser, A. J., Melis, C., et al. 2013, AJ, 145, 71

Kirkpatrick, J. D., Looper, D. L., Burgasser, A. J., et al. 2010, ApJS, 190, 100

Konopacky, Q. M., Ghez, A. M., Barman, T. S., et al. 2010, ApJ, 711, 1087

Kraus, A. L., \& Hillenbrand, L. A. 2012, ApJ, 757, 141

Liu, M. C., Dupuy, T. J., \& Leggett, S. K. 2010, ApJ, 722, 311

Looper, D. L., Gelino, C. R., Burgasser, A. J., \& Kirkpatrick, J. D. 2008a, ApJ, 685,1183

Looper, D. L., Kirkpatrick, J. D., \& Burgasser, A. J. 2007, AJ, 134, 1162

Looper, D. L., Kirkpatrick, J. D., Cutri, R. M., et al. 2008b, ApJ, 686, 528

Manjavacas, E., Goldman, B., Reffert, S., \& Henning, T. 2013, A\&A, 560, A52

Metchev, S. A., Kirkpatrick, J. D., Berriman, G. B., \& Looper, D. 2008, ApJ, 676, 1281

Metropolis, N., Rosenbluth, A. W., Rosenbluth, M. N., Teller, A. H., \& Teller, E. 1953, JChPh, 21, 1087

Monet, D. G., Levine, S. E., Canzian, B., et al. 2003, AJ, 125, 984

Pravdo, S. H., Shaklan, S. B., Wiktorowicz, S. J., et al. 2006, ApJ, 649, 389

Radigan, J., Jayawardhana, R., Lafrenière, D., et al. 2012, ApJ, 750, 105

Raghavan, D., McAlister, H. A., Henry, T. J., et al. 2010, ApJS, 190, 1

Rayner, J. T., Toomey, D. W., Onaka, P. M., et al. 2003, PASP, 115, 362

Reid, I. N., Cruz, K. L., Kirkpatrick, J. D., et al. 2008, AJ, 136, 1290

Saumon, D., \& Marley, M. S. 2008, ApJ, 689, 1327

Scholz, R.-D. 2014, A\&A, 561, A113

Silvestri, N. M., Lemagie, M. P., Hawley, S. L., et al. 2007, AJ, 134, 741

Simons, D. A., \& Tokunaga, A. 2002, PASP, 114, 169

Stephens, D. C., Leggett, S. K., Cushing, M. C., et al. 2009, ApJ, 702, 154

Stumpf, M. B., Geißler, K., Bouy, H., et al. 2011, A\&A, 525, A123

Tokunaga, A. T., Simons, D. A., \& Vacca, W. D. 2002, PASP, 114, 180

van Dam, M. A., Bouchez, A. H., Le Mignant, D., et al. 2006, PASP, 118, 310 van den Bos, W. H. 1927, BAN, 3, 261

Wizinowich, P. L., Le Mignant, D., Bouchez, A. H., et al. 2006, PASP, 118,297 